\title{
Plant Resources Utilization among Different Ethnic Groups of Ladakh in Trans-Himalayan Region
}

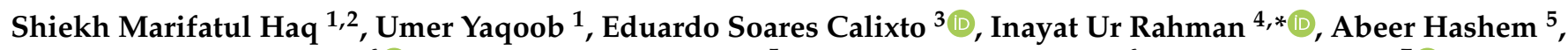 \\ Elsayed Fathi Abd_Allah ${ }^{6}\left({ }^{\circ}\right.$, Maha Abdullah Alakeel ${ }^{5}$, Abdulaziz A. Alqarawi ${ }^{6}$, Mohnad Abdalla ${ }^{7}(\mathbb{D}$, \\ Musheerul Hassan $\left.{ }^{8}{ }^{(}\right)$, Rainer W. Bussmann $\left.{ }^{9}{ }^{(}\right)$, Arshad Mehmood Abbasi ${ }^{10}\left({ }^{\circ}\right.$, Sami Ur Rahman ${ }^{11}$ and \\ Farhana Ijaz ${ }^{4}$
}

check for updates

Citation: Haq, S.M.; Yaqoob, U.; Calixto, E.S.; Rahman, I.U.; Hashem, A.; Abd_Allah, E.F.; Alakeel, M.A.; Alqarawi, A.A.; Abdalla, M.; Hassan, M.; et al. Plant Resources Utilization among Different Ethnic Groups of Ladakh in Trans-Himalayan Region. Biology 2021, 10, 827. https:// doi.org/10.3390/biology10090827

Academic Editor:

Panayiotis Dimitrakopoulos

Received: 23 June 2021

Accepted: 9 August 2021

Published: 26 August 2021

Publisher's Note: MDPI stays neutral with regard to jurisdictional claims in published maps and institutional affiliations.

Copyright: (c) 2021 by the authors. Licensee MDPI, Basel, Switzerland. This article is an open access article distributed under the terms and conditions of the Creative Commons Attribution (CC BY) license (https:// creativecommons.org/licenses/by/ $4.0 /)$.
1 Department of Botany, University of Kashmir Srinagar, Srinagar 190006, India; marifat.edu.17@gmail.com (S.M.H.); umer.scholar@kashmiruniversity.net (U.Y.)

2 Wildlife Crime Control Division, Wildlife Trust of India, Noida 201301, India

3 Institute of Biology, University of São Paulo, São Paulo 05315-970, Brazil; calixtos.edu@gmail.com

4 Department of Botany, Hazara University, Mansehra 21300, Pakistan; fbotany@yahoo.com

5 Botany and Microbiology Department, College of Science, King Saud University, Riyadh 11451, Saudi Arabia; habeer@ksu.edu.sa (A.H.); 441203162@student.ksu.edu.sa (M.A.A.)

6 Department of Plant Production, College of Food and Agriculture Science, King Saud University, Riyadh 11451, Saudi Arabia; eabdallah@ksu.edu.sa (E.F.A.); alqarwy@ksu.edu.sa (A.A.A.)

7 Key Laboratory of Chemical Biology (Ministry of Education), Department of Pharmaceutics, School of Pharmaceutical Sciences, Cheeloo College of Medicine, Shandong University, 44 Cultural West Road, Jinan 250012, China; mohnadabdalla200@gmail.com

8 Clybay Research Private Limited, Bangalore 560114, India; musheer@clybay.com

9 Department of Ethnobotany, Institute of Botany, Ilia State University, 1 Botanical Street, Tbilisi 0105, Georgia; rainer.bussmann@iliauni.edu.ge

10 Department of Environmental Sciences, Abbottabad Campus, COMSATS University Islamabad Islamabad 22060, Pakistan; amabbasi@cuiatd.edu.pk

11 Nawaz Sharif Kidney Teaching Hospital and Postgraduate Institute Manglawar, Swat 19200, Pakistan; drsamiurrahman.swat@gmail.com

* Correspondence: hajibotanist@outlook.com

Simple Summary: Indigenous communities are a large resource of increasingly endangered, traditionally used medicinal plants and the associated ecological knowledge, which needs to be documented quickly as the base to establish sustainable livelihoods and healthcare systems. Through the interaction of indigenous knowledge, biodiversity, and the surrounding environment, these communities have developed their livelihoods over time. In this study, we tried to obtain an in-depth understanding of ethnomedicinal, cultural, and ritual perspectives on plant diversity in the Ladakh region and evaluated how the wild flora of Ladakh could improve local livelihoods and alleviate poverty. By surveying and applying open- and close-ended semi-structured interviews and group discussions in three communities, we documented 105 ethnobotanically important plants belonging to 39 families. The Balti and Brokpa ethnic groups showed greater similarity, whereas the least overlap in plant use was observed between Beda and Brokpa. Plants common to all cultures were mostly used for medicinal applications, while some were also used for religious purposes in the two major religions (Islam and Buddhism). A total of 37 species were shared by all cultures (Balti, Brokpa, and Beda). The cluster analysis elucidated three major clusters of different ethnobotanical usage. The first cluster included food and medicinal plants, the second included clusters of dye and flavor plants, and the third included plants used for fragrance, oil, fuel wood, and fodder. Plant parts were collected based on their availability in distinct pheno-phases according to the Tibetan traditional calendar. This study's findings revealed that plants provide tangible economic benefits to indigenous communities, in addition to aiding in the treatment of various ailments. Sustainable use and management of wild resources can help improve livelihoods and food security and alleviate poverty.

Abstract: The nomadic pastoral indigenous communities of the Ladakhi people share roots with Tibetan culture in terms of food, clothing, religion, festivals, and habits, and rely widely on plant resources for survival and livelihood. This survey was conducted during 2019-2021 to document 
the indigenous knowledge about plant resources of the Balti, Beda, and Brokpa communities of the Ladakh region, trans-Himalayas. Open- and close-ended semi-structured interviews $(\mathrm{N}=184)$ and group discussions $(\mathrm{N}=17)$ were used to collect the data. Quantitative data was further analyzed using various statistical tools. A total of 105 plant species belonging to 82 genera and 39 families were used as medicine, fuel wood, fragrance, oil, food, flavor, fodder, decoration, and dye. Among these, medicinal use was most prevalent, with $70 \%$ of use reports, followed by fodder and fuel wood. Leaves $(27 \%)$ were the most preferred plant part used, followed by roots and flowers. The principal component analysis revealed five clusters of ethnobotanical usage, i.e., food, medicine, fuel wood, fodder, and fragrance, oil, dye, and flavor. The maximum number of plant species used was reported by the Brokpa, while the Beda reported the minimum number of plant species uses. Delphinium brunonianum, Waldheimia tomentosa, and Juniperus indica played a significant role in the cultural and religious ritual aspects, whereas Allium przewalskianum, Waldheimia tomentosa, Juniperus indica, and Hippophae rhamnoides were commonly used as a livelihood source among Ladakhi communities. The local people collected most plants $(65 \%)$ for self-consumption, while the rest $(35 \%)$ were sold in markets as a source of income. The sustainable utilization and management of plant resources by local people is a strategy to boost livelihoods and food security and alleviate poverty.

Keywords: biodiversity; ethnobotanical uses; plant resource; Ladakh; chord diagram; trans-Himalayas

\section{Introduction}

Humanity has always been dependent on ecosystem services [1]. Plant biodiversity throughout the globe has been providing these ecosystem services in terms of both economy and culture [2], including food and fodder for humans and their livestock, timber, firewood, and herbal remedies for treating various ailments [3]. Many plant resources have cultural importance, e.g., in education, in religion, as totems, for aesthetics, etc., and are important for socio-economic and industrial activities [4,5].

Indigenous communities have developed broad ecological knowledge and are, often, still dependent on wild plants for food, fodder, medicines, and other purposes. The focus on traditional plant foraging is especially vital in remote tribal areas in comprehending its role in the sustainability of food systems and for the promotion and discovery of novel local gastronomies [6]. Ethnomedical practices have resulted in the development of traditional medicinal systems such as Unani, Siddha, Sowa-Rigpa, and Ayurveda and are also part of many even allopathic drugs [7]. The population of developing countries is especially reliant on these traditional plant-based medicines due to the lack of modern health facilities [8]. Ethnobiological field studies have widely shown that indigenous communities represent a significant reservoir of disappearing folk plants and ecological knowledge, which needs to be immediately documented to develop sustainable food and healthcare systems [9]. Wild plants play an imperative role in the livelihood of tribal people [10]. Over the years, traditional knowledge has resulted in the development of systems providing livelihood to the indigenous communities. These sustainable livelihoods are developed over the years through the interaction of indigenous knowledge, biodiversity, and the surrounding environment [11]. Traditional knowledge has also been proved to be effective in the conservation of biological resources, which are important stakeholders in conserving biodiversity. This complex relationship is difficult to manage by outside experts. Thus, to conserve the diversity of different plants used by indigenous communities, it is imperative to involve indigenous communities in conserving them as they know how the different interaction factors work with each other [11].

Ladakh has a rich history of medicinal and wild food plants, but limited studies on the utilization of wild plant species that could help in identifying novel and potential sources of medicines, food, and other plant products have been carried out to document the associated traditional knowledge. The Sowa-Rigpa herbal medicine system is considered the oldest codified healthcare system known to humanity [12]. The conservation of the records of 
this valuable knowledge is as imperative as medicinal plant conservation [13]. Our study quantified the indigenous ethnomedicinal uses of plants and assessed the distribution, composition, conservation, and trade of medicinal plants of Ladakh. The main objectives of the fieldwork were (1) to gain an in-depth understanding of ethnomedicinal, cultural, and ritual perspectives of plant diversity in the Ladakh region and (2) to evaluate how the wild flora of Ladakh could boost up livelihood and food security and support in poverty alleviation. Various studies have reported medicinal and other ethno uses of plants from Ladakh [14,15]; however, the local population in our study area (Balti, Beda, and Brokpa) has never participated in an ethnobotanical study.

\section{Materials and Methods}

\subsection{Study Area}

The Ladakh region in the trans-Himalayas of the Indian Himalayan Region (IHR) is one of the highest plateaus in the world. The study area is part of the previously northern Jammu and Kashmir state and is located at $34^{\circ} 12^{\prime} 34.25^{\prime \prime} \mathrm{N}$ and $77^{\circ} 36^{\prime} 54.40^{\prime \prime} \mathrm{E}$ (Figure 1). Ladakh covers less than half of the landmass of the state and is surrounded by Tibet (China) to the north and northeast, Gilgit-Baltistan (Pakistan) to the northwest, and Himachal Pradesh to the west. The total area of Ladakh is $59,146 \mathrm{~km}^{2}$, which as per the 2010 census had a population of 290,492 , with a population density of 4.91 people $/ \mathrm{km}^{2}$, representing the lowest density in the country. Administratively, Ladakh has two districts: Kargil, with $14,036 \mathrm{~km}^{2}$ area and about 143,388 inhabitants, and Leh district $\left(45,110 \mathrm{~km}^{2}, 147,104\right.$ inhabitants) [16]. The largest town in Ladakh is Leh, located at an altitude of $3000 \mathrm{~m}$, followed by Kargil at $2676 \mathrm{~m}$, each of which headquarters a district. The area is cold and dry, with an annual rainfall of less than $120 \mathrm{~mm}$. In winter, the entire area is covered with heavy snow. Most of the settlements are in the river valleys. The flora of Ladakh is a rich source of aromatic and medicinal plants and may be grouped into three broad classes—arid vegetation, alpine mesophytes, and oasitic or riparian vegetation [17].

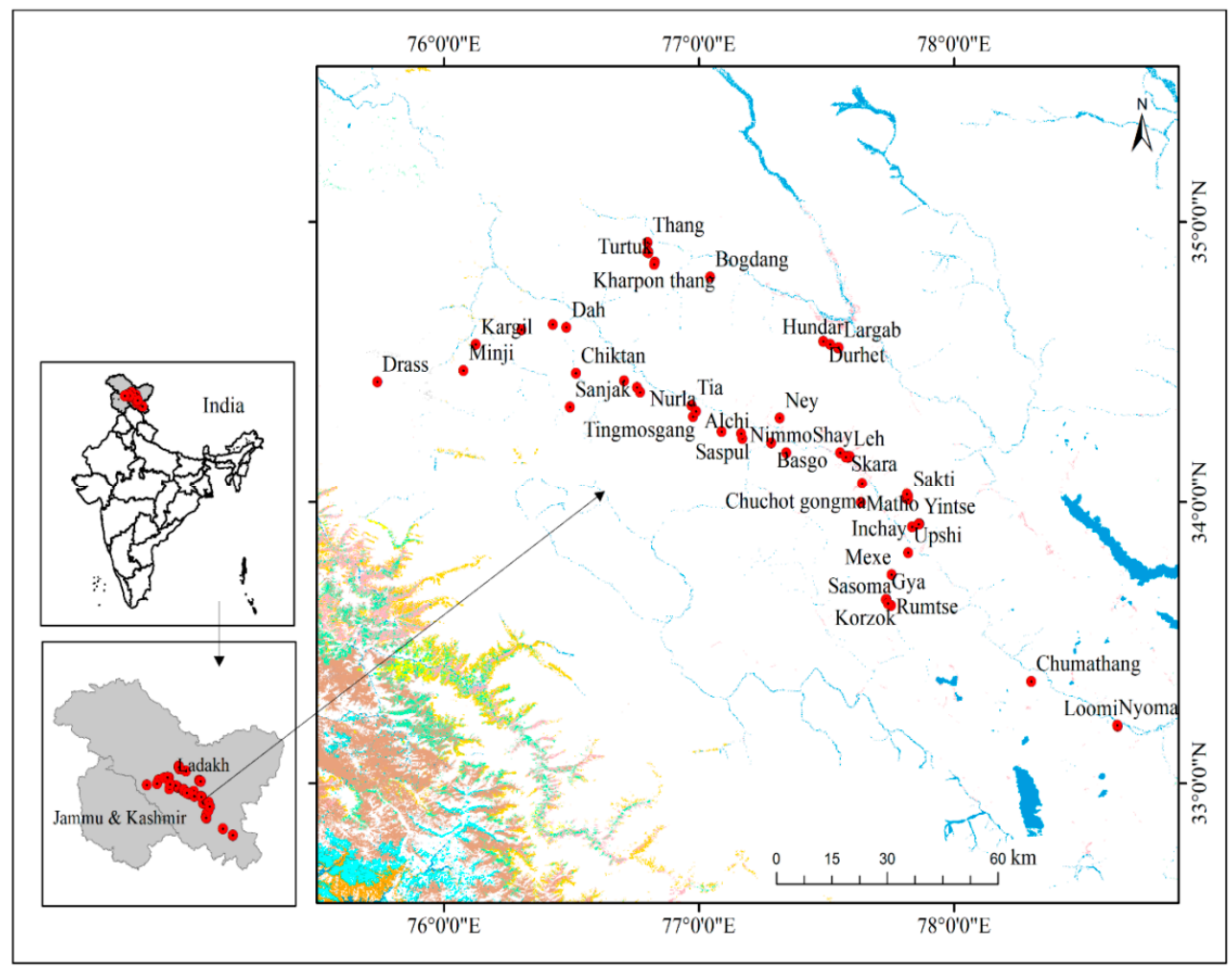

Figure 1. Map of the study area showing the sampling sites $(n=54)$ in the Ladakh trans-Himalayan region, India. A global positioning system (GPS Garmin map76cs) was used to record the altitude as well as geo-coordinates of the sampled sites. 
Leh comprises the Indus, Shyok, and Nubra river valleys, while Kargil includes the Suru, Dras, and Zanskar river valleys. The Nubra, Shyok and Indus River valleys are the most populated areas of the district Leh. The main ethnic groups include Balti, Beda, and Brokpa, all living across both divisions. The Balti tribe is originally of Tibetan descent and mainly found in the Kargil region, although a small population also lives in Leh. The spoken language is known as Balti. The Beda are a unique tribe famous for their musicianship (traditional occupation) and scattered in Ladakh. They predominantly follow the Muslim faith. The Brokpa (people living in mountains) are a small community of Dard people found in Leh and Kargil. Those living in Leh are Buddhists and the inhabitants of Kargil are Muslims. A small number of people have embraced Hinduism. Their language is known as Brokstat.

\subsection{Data Collection}

The present study was carried out over the period 2019-2021 in the Balti, Beda, and Brokpa ethnic groups. Information was gathered through open- and close-ended semistructured interviews $(\mathrm{N}=184)$ and group discussions $(\mathrm{N}=17)$ following [16]. Participants $(\mathrm{N}=269$ ) included $77.32 \%$ men and $22.67 \%$ women, of which $7.80 \%$ were Amchis (traditional doctors), herders (8.92\%), hunters (5.94\%), shopkeepers $(4.83 \%)$, farmers $(30.48 \%)$, daily wage laborers $(7.80 \%)$, hotel owners $(5.57 \%)$, museum owners $(0.74 \%)$, housewives $(19.33 \%)$, and government employees (8.55\%). Interviews and discussions focused on the ethnobotanical use of local plant resources as medicine, food, fodder, fuel wood, fragrance, dye, decoration, flavor, and oil, including information on harvesting time and season. The ethnicity of the participants and the language information given here are not disclosed, based on mutual agreement as stipulated under the Nagoya Protocol. The code of ethics of the International Society of Ethnobiology was followed [18]. Additionally, one person from each indigenous community, who was well familiar with the traditions and norms of the community, was taken as a guide during all the field surveys. Information was gathered about key plant species used for cultural, religious, and ritual beliefs, and compared to $[13,19]$.

Plant specimens were collected from different sites during the field survey and were properly coded/tagged. Specimens were identified with the help of taxonomists at the CBT Lab, University of Kashmir, Srinagar (J\&K), by comparing with herbarium specimens at the KASH herbarium and local floras [20]. The nomenclature and botanical families of all the specimens were further authenticated using www.plantsoftheworldonline.org/ (accessed on 28 November 2020).

\subsection{Data Analysis}

Analyses of the ethnobotanical uses of plant species were carried out using cluster analysis. We used absence/presence data to show the distribution of the species, clustering species with similar ethnobotanical uses using PAST software ver. 3.14. Sørensen's (Bray-Curtis) distance similarity coefficient, based on presence/absence data, was used to identify significant differences among diverse ethnobotanical uses and plant species [21,22]. Principal component analysis (PCA) was performed to visualize provisioning services and plant parts used, using the package "vegan" [23] in the software R 4.0.0 [24]. To evaluate whether there was a difference in the number of plant parts used, we used a generalized linear model (GLM) with binomial distribution, followed by the likelihood-ratio test. The contribution of different plant parts used was displayed in chord diagrams using the circlize package [25] in R software 3.6.1 [23]. The Venn diagram was created using Bioinformatics \& Evolutionary Genomics software (http:/ / bioinformatics.psb.ugent.be/cgi-bin/ liste/Venn/calculate_venn.htpl (accessed on 21 January 2021)).

\section{Results and Discussion}

The results of the study revealed that the local population of the study area is still a rich source of herbal medicines and traditional knowledge. Furthermore, the current work 
evidently indicates the close connection between the local population and provisioning ecosystem services of plants.

\subsection{Demographic Details of Respondents and Vegetation Composition}

In the present study, the respondents represented a diverse array of professional groups, including daily wage laborers, farmers, government employees, herders, hotel owners, hunters, homemakers, museum owners, shopkeepers, and traditional doctors (Amchis), across three ethnic groups, i.e., Beda $(\mathrm{N}=63)$, Balti $(\mathrm{N}=91)$, and Brokpa $(\mathrm{N}=115$; Table 1). Among the 269 respondents, $77.3 \%$ were men, and the remaining $22.7 \%$ were women. A possible reason for the smaller number of female informants is that they are confined to their homes due to cultural restrictions [26,27]. Most of the informants ( $48 \%$ ) were $46-65$ years old, followed by $66-88$ (40\%) and $25-45(12 \%)$. More than half of the respondents were without formal education (65\%; Table 1$)$. We noticed that older people hold more traditional knowledge than younger people in this area, a fact also reported in earlier studies [28]. As in other parts of the Himalayan region, ethnic knowledge about the uses of different therapeutic plants was decreasing in the younger people of the study area, which may be ascribed to little interest shown by the younger generation in inheriting and using ethnomedical practices [29]. In addition, the illiterate population was found to have more ethnomedical information, which may be ascribed to the fact that educated participants are expected to be exposed to the developed world and mostly rely on modern medicines instead of traditional ones [30]. During the survey, it was noticed that the population in rural areas also had more knowledge of natural resources compared to urban populations.

Table 1. Demographic details of respondents interviewed in the present study.

\begin{tabular}{|c|c|c|}
\hline Professional Groups Interviewed & Professional Group & Number \\
\hline & Farmers & 82 \\
\hline & Housewives & 52 \\
\hline & Herders & 24 \\
\hline & Govt. employees & 23 \\
\hline & Amchis & 21 \\
\hline & Daily wage laborers & 21 \\
\hline & Shopkeepers & 13 \\
\hline & Hunters & 16 \\
\hline & Hotel owners & 15 \\
\hline & Museum owners & 2 \\
\hline \multirow{2}{*}{ Gender } & Male & 208 \\
\hline & Female & 61 \\
\hline \multirow{3}{*}{ Ethnic group } & Balti & 91 \\
\hline & Beda & 63 \\
\hline & Brokpa & 115 \\
\hline \multirow{3}{*}{ Age group } & $25-45$ & 33 \\
\hline & $46-65$ & 128 \\
\hline & $65-88$ & 106 \\
\hline \multirow{6}{*}{ Education qualification } & Without formal education & 174 \\
\hline & 5 th pass & 37 \\
\hline & 8th pass & 21 \\
\hline & 10th pass & 15 \\
\hline & 12th pass & 14 \\
\hline & Graduate and above & 8 \\
\hline
\end{tabular}


In this study, we documented 105 ethnobotanically important plants belonging to 82 genera and 39 families (Table 2). The respective uses, i.e., medicine, fuel wood, fragrance, oil, food, flavor fodder, decoration, and dye, are presented in Figure 2. The number of plant species recorded in the study area was close to those documented by earlier ethnobotanical studies in other parts of the Himalayan region. Bhattarai et al. [26] and Ambu et al. [31] reported 121 and 116 species from the trans-Himalayan region of Nepal. Awan et al. [32], Mulk et al. [33], and Ajaib et al. [34] reported a total of 102, 101, and 100 plant species, respectively, from the Western Himalayas of Pakistan. Similar results were reported by Rana and Rawat [35], Kayani et al. [36], and Haider and Qaiser [37] in the Himalayan region. During the field work, it was noted that medicinal plant richness decreased with altitude, while the percentage of use reports of medicinal plants also gradually increased with altitude. This may be a result of the preference given by the local population to medicinal plants from higher-altitude areas. Lone et al. [38] also reported similar results from the Bandipora district of Jammu and Kashmir.

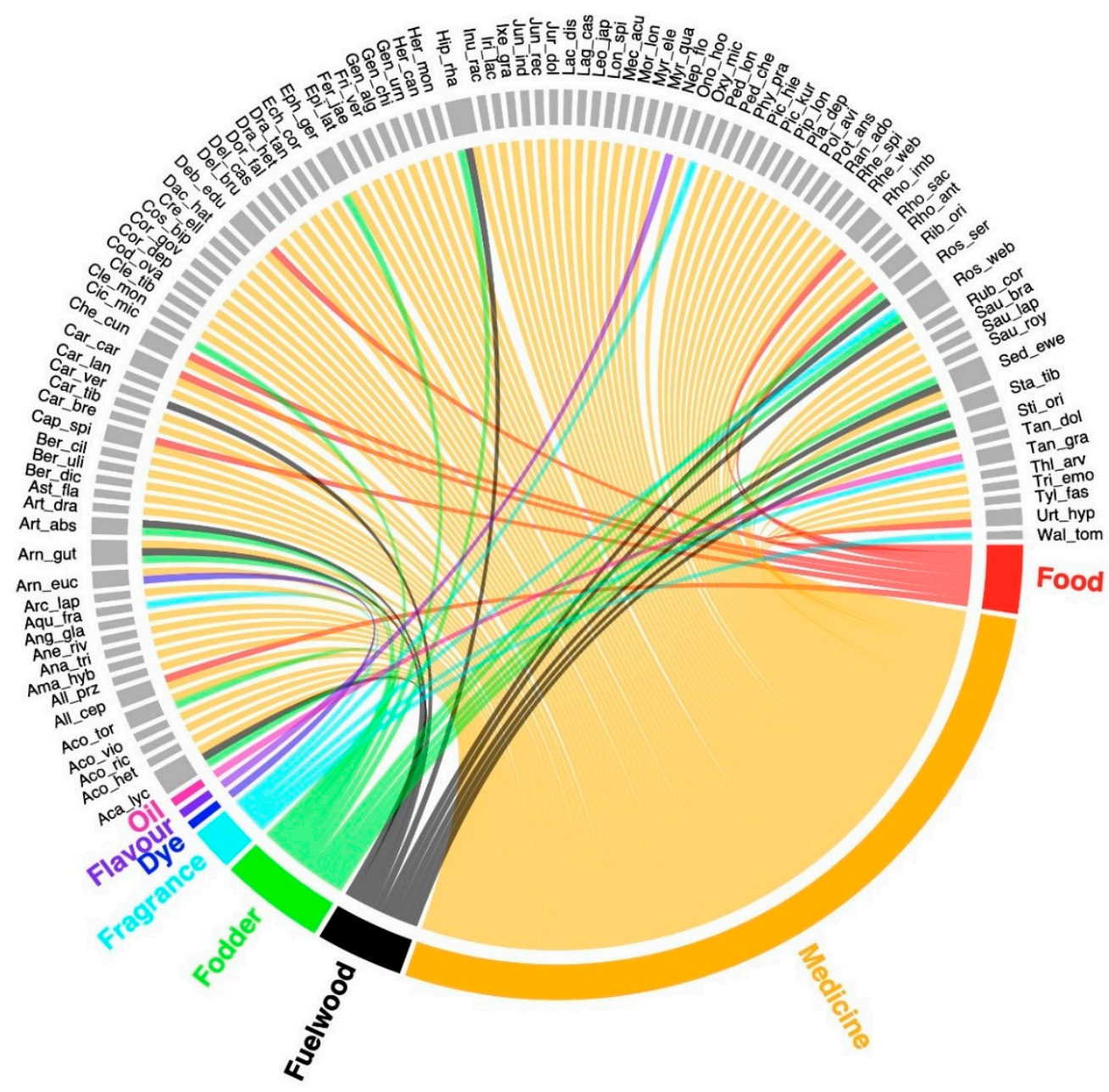

Figure 2. Plant species distribution (105 species) according to plant usage in the Ladakh transHimalayan region, India. 
Table 2. List of plant species with their ethnomedicinal usage in the Ladakh trans-Himalayan region, India.

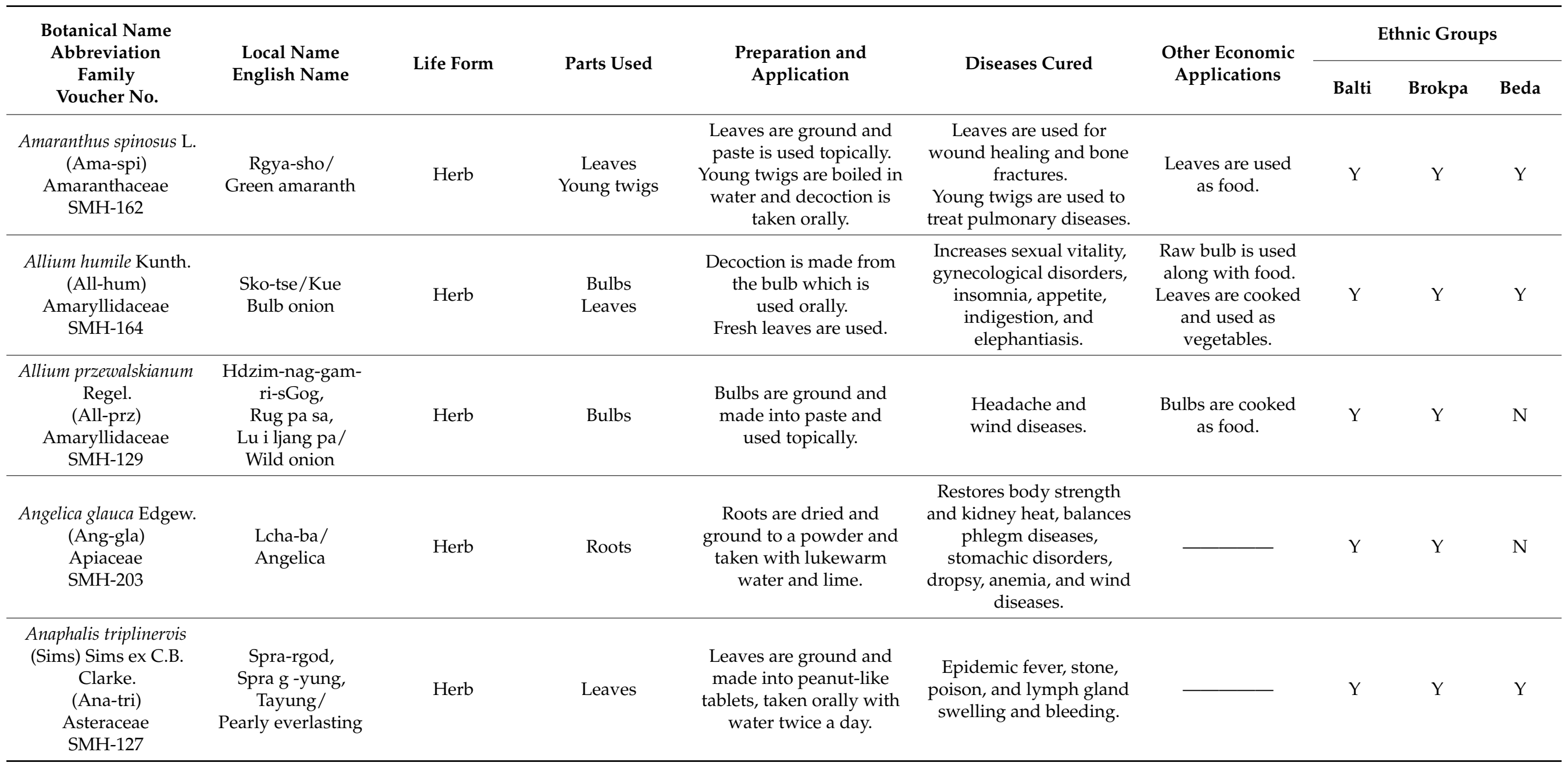


Table 2. Cont.

\begin{tabular}{|c|c|c|c|c|c|c|c|c|c|}
\hline \multirow{2}{*}{$\begin{array}{c}\text { Botanical Name } \\
\text { Abbreviation } \\
\text { Family } \\
\text { Voucher No. }\end{array}$} & \multirow{2}{*}{$\begin{array}{l}\text { Local Name } \\
\text { English Name }\end{array}$} & \multirow{2}{*}{ Life Form } & \multirow{2}{*}{ Parts Used } & \multirow{2}{*}{$\begin{array}{l}\text { Preparation and } \\
\text { Application }\end{array}$} & \multirow{2}{*}{ Diseases Cured } & \multirow{2}{*}{$\begin{array}{c}\text { Other Economic } \\
\text { Applications }\end{array}$} & \multicolumn{3}{|c|}{ Ethnic Groups } \\
\hline & & & & & & & Balti & Brokpa & Beda \\
\hline $\begin{array}{l}\text { Arctium lappa L. } \\
\text { (Arc-lap) } \\
\text { Asteraceae } \\
\text { SMH-185 }\end{array}$ & $\begin{array}{l}\text { Byi-bzung/ } \\
\text { Greater burdock }\end{array}$ & Herb & $\begin{array}{l}\text { Roots } \\
\text { Seeds } \\
\text { Leaves }\end{array}$ & $\begin{array}{l}\text { Decoction is made from } \\
\text { roots and taken orally. } \\
\text { Seeds and leaves are } \\
\text { ground to a fine powder, } \\
\text { which is taken with water } \\
\text { for a week. } \\
\text { Leaves are ground to a } \\
\text { fine powder and taken } \\
\text { orally with water. }\end{array}$ & $\begin{array}{l}\text { Roots are believed to have } \\
\text { anticancer potential and } \\
\text { used against uterus tumors } \\
\text { and urinary bladder cysts. } \\
\text { Seeds are used to cure } \\
\text { burning urination. } \\
\text { Leaves are used for nerve } \\
\text { disorders and unripe } \\
\text { epidemic fever. }\end{array}$ & $\longrightarrow$ & $\mathrm{N}$ & $\mathrm{Y}$ & $\mathrm{N}$ \\
\hline $\begin{array}{c}\text { Artemisia absinthium L. } \\
\text { (Art-abs) } \\
\text { Asteraceae } \\
\text { SMH-125 }\end{array}$ & $\begin{array}{l}\text { Bur-tse, } \\
\text { Khan dkar, } \\
\text { Dpa bo nyid/ } \\
\text { Wormwood }\end{array}$ & Herb & Leaves & $\begin{array}{l}\text { Tea is obtained by boiling } \\
\text { the dry and fresh leaves } \\
\text { for more than half an } \\
\text { hour and taken orally. }\end{array}$ & $\begin{array}{l}\text { Analgesic during labor } \\
\text { pain, leukemia, } \\
\text { and sclerosis. }\end{array}$ & - & $\mathrm{Y}$ & $\mathrm{Y}$ & $\mathrm{N}$ \\
\hline $\begin{array}{c}\text { Artemisia dracunculus } \\
\text { L. } \\
\text { (Art-dra) } \\
\text { Asteraceae } \\
\text { SMH-131 }\end{array}$ & $\begin{array}{l}\text { Tse-phat, } \\
\text { tshar-bong/ } \\
\text { Tarragon }\end{array}$ & Herb & Leaves & $\begin{array}{l}\text { Ground fresh leaves are } \\
\text { made into small tablets } \\
\text { and taken orally. }\end{array}$ & $\begin{array}{l}\text { Digestive disorders, } \\
\text { toothache, pharyngitis, } \\
\text { pulmonary diseases, and } \\
\text { swelling due to } \\
\text { hot disorders. }\end{array}$ & $\longrightarrow$ & $\mathrm{Y}$ & $\mathrm{Y}$ & $\mathrm{N}$ \\
\hline $\begin{array}{c}\text { Aster flaccidus Bunge. } \\
\text { (Ast-fla) } \\
\text { Asteraceae } \\
\text { SMH-103 }\end{array}$ & $\begin{array}{l}\text { Me-tok-lug-mig, } \\
\text { Nia-Mentok/ } \\
\text { Alpine aster }\end{array}$ & Herb & $\begin{array}{l}\text { Flowers } \\
\text { Leaves }\end{array}$ & $\begin{array}{l}\text { Decoction is made from } \\
\text { dry and fresh flowers, } \\
\text { taken orally. } \\
\text { Paste is made from leaves } \\
\text { and applied topically. }\end{array}$ & $\begin{array}{c}\text { Flowers are used against } \\
\text { poisoning, epidemic fever, } \\
\text { infectious cold and cough, } \\
\text { spasms of tendons and } \\
\text { ligaments. } \\
\text { Leaves are used to treat } \\
\text { drying pus and blood of } \\
\text { wounds, and blisters. }\end{array}$ & $\begin{array}{l}\text { Flowers are used } \\
\text { as ornaments. }\end{array}$ & $\mathrm{Y}$ & $\mathrm{Y}$ & $\mathrm{N}$ \\
\hline $\begin{array}{c}\text { Acantholimon } \\
\text { lycopodioides } \\
\text { (Girard) Boiss. } \\
\text { (Aca-lyc) } \\
\text { Plumbaginaceae } \\
\text { SMH-128 }\end{array}$ & $\begin{array}{l}\text { Long-zay/ } \\
\text { Prickly thrift }\end{array}$ & Herb & Leaves & $\begin{array}{l}\text { Dried and fresh leaves } \\
\text { are boiled in water to } \\
\text { make decoction, which is } \\
\text { taken orally twice a day. }\end{array}$ & Cardiac disorders. & $\begin{array}{l}\text { Litter in the form } \\
\text { of dry leaves } \\
\text { serves as fuel. }\end{array}$ & Y & Y & $\mathrm{Y}$ \\
\hline
\end{tabular}


Table 2. Cont.

\begin{tabular}{|c|c|c|c|c|c|c|c|c|c|}
\hline \multirow{2}{*}{$\begin{array}{c}\text { Botanical Name } \\
\text { Abbreviation } \\
\text { Family } \\
\text { Voucher No. }\end{array}$} & \multirow{2}{*}{$\begin{array}{l}\text { Local Name } \\
\text { English Name }\end{array}$} & \multirow{2}{*}{ Life Form } & \multirow{2}{*}{ Parts Used } & \multirow{2}{*}{$\begin{array}{l}\text { Preparation and } \\
\text { Application }\end{array}$} & \multirow{2}{*}{ Diseases Cured } & \multirow{2}{*}{$\begin{array}{l}\text { Other Economic } \\
\text { Applications }\end{array}$} & \multicolumn{3}{|c|}{ Ethnic Groups } \\
\hline & & & & & & & Balti & Brokpa & Beda \\
\hline $\begin{array}{c}\text { Aconogonum tortuosum } \\
\text { (D. Don) H. Hara. } \\
\text { (Aco-tor) } \\
\text { Polygonaceae } \\
\text { SMH-102 }\end{array}$ & $\begin{array}{c}\text { Rnyalo, } \\
\text { Chu rtsi, } \\
\text { Skyu ru mu ru/ } \\
\text { Twisted } \\
\text { Knotweed }\end{array}$ & Herb & Roots & $\begin{array}{l}\text { Roots are boiled in water } \\
\text { and then taken orally after } \\
\text { more than two hours. }\end{array}$ & $\begin{array}{l}\text { Black motion, dysentery, } \\
\text { diarrhea, lumbar pain } \\
\text { due to delivery. }\end{array}$ & $\longrightarrow$ & $\mathrm{Y}$ & $\mathrm{Y}$ & $\mathrm{N}$ \\
\hline $\begin{array}{c}\text { Aconitum heterophyllum } \\
\text { Wall. ex Royle. } \\
\text { (Aco-het) } \\
\text { Ranunculaceae } \\
\text { SMH-195 }\end{array}$ & $\begin{array}{l}\text { Boona-karpo/ } \\
\text { Indian atees }\end{array}$ & Herb & Roots & $\begin{array}{l}\text { Paste is obtained via } \\
\text { grinding the roots and } \\
\text { applied topically. }\end{array}$ & $\begin{array}{l}\text { Useful in toothache and } \\
\text { headache. }\end{array}$ & & Y & Y & $\mathrm{N}$ \\
\hline $\begin{array}{c}\text { Aconitum } \\
\text { richardsonianum } \\
\text { Lauener. } \\
\text { (Aco-ric) } \\
\text { Ranunculaceae } \\
\text { SMH-187 }\end{array}$ & $\begin{array}{l}\text { Bong-nag-lo- } \\
\text { pohra/ } \\
\text { Monkshood }\end{array}$ & Herb & Roots & $\begin{array}{l}\text { Dried roots are ground to a } \\
\text { fine powder and taken with } \\
\text { lukewarm water orally. }\end{array}$ & $\begin{array}{l}\text { Common cold, } \\
\text { pneumonia, laryngitis, } \\
\text { croup, and asthma, } \\
\text { inflammation, and high } \\
\text { blood pressure. }\end{array}$ & - & Y & $\mathrm{Y}$ & $\mathrm{N}$ \\
\hline $\begin{array}{l}\text { Aconitum violaceum } \\
\text { Jacquem. ex Stapf. } \\
\text { (Aco-vio) } \\
\text { Ranunculaceae } \\
\text { SMH-163 }\end{array}$ & $\begin{array}{c}\text { Boona-nagpo/ } \\
\text { Violet monkshood }\end{array}$ & Herb & Roots & $\begin{array}{l}\text { Roots are dried in shade } \\
\text { and ground to a powder, } \\
\text { taken with lukewarm } \\
\text { water. }\end{array}$ & $\begin{array}{l}\text { Cold, cough, asthma, } \\
\text { fever, and gastric } \\
\text { problems. }\end{array}$ & - & Y & $\mathrm{Y}$ & $\mathrm{N}$ \\
\hline $\begin{array}{c}\text { Anemone rivularis } \\
\text { Buch.-Ham. ex DC. } \\
\text { (Ane-riv) } \\
\text { Ranunculaceae } \\
\text { SMH-186 }\end{array}$ & $\begin{array}{l}\text { Srub-ka, } \\
\text { Sngo srub/ } \\
\text { Riverside } \\
\text { windflower }\end{array}$ & Herb & Roots & $\begin{array}{l}\text { Dried roots are ground to } \\
\text { powder and taken with } \\
\text { boiled water orally. }\end{array}$ & $\begin{array}{l}\text { Severe body pain, } \\
\text { stomach heat, snake } \\
\text { poison, tumors and } \\
\text { drying serous fluids. }\end{array}$ & & Y & Y & Y \\
\hline $\begin{array}{l}\text { Aquilegia fragrans Benth. } \\
\text { (Aqu-fra) } \\
\text { Ranunculaceae } \\
\text { SMH-130 }\end{array}$ & $\begin{array}{l}\text { Doftakleja/ } \\
\text { Fragrant } \\
\text { columbine }\end{array}$ & Herb & Flowers & Fresh flowers are used. & - & $\begin{array}{l}\text { Flowers are used } \\
\text { for ornamental } \\
\text { purposes. }\end{array}$ & Y & Y & $\mathrm{Y}$ \\
\hline
\end{tabular}


Table 2. Cont

\begin{tabular}{|c|c|c|c|c|c|c|c|c|c|}
\hline \multirow{2}{*}{$\begin{array}{l}\text { Botanical Name } \\
\text { Abbreviation } \\
\text { Family } \\
\text { Voucher No. }\end{array}$} & \multirow{2}{*}{$\begin{array}{l}\text { Local Name } \\
\text { English Name }\end{array}$} & \multirow{2}{*}{ Life Form } & \multirow{2}{*}{ Parts Used } & \multirow{2}{*}{$\begin{array}{c}\text { Preparation and } \\
\text { Application }\end{array}$} & \multirow{2}{*}{ Diseases Cured } & \multirow{2}{*}{$\begin{array}{l}\text { Other Economic } \\
\text { Applications }\end{array}$} & \multicolumn{3}{|c|}{ Ethnic Groups } \\
\hline & & & & & & & Balti & Brokpa & Beda \\
\hline $\begin{array}{l}\text { Arnebia euchroma } \\
\text { (Royle) I.M.Johnst. } \\
\text { (Arn-euc) } \\
\text { Boraginaceae } \\
\text { SMH-126 }\end{array}$ & $\begin{array}{c}\text { Bri-mok, } \\
\text { Demok/ } \\
\text { Pink arnebia }\end{array}$ & Herb & Roots & $\begin{array}{l}\text { Roots are shade-dried } \\
\text { and decoction is made } \\
\text { from ground roots, taken } \\
\text { orally for a week. }\end{array}$ & $\begin{array}{l}\text { Blood purifier, cold, } \\
\text { cough, lung, and } \\
\text { pulmonary problems. }\end{array}$ & $\begin{array}{l}\text { Roots are used as } \\
\text { coloring dishes } \\
\text { and sweets. }\end{array}$ & $\mathrm{Y}$ & $\mathrm{Y}$ & $\mathrm{Y}$ \\
\hline $\begin{array}{c}\text { Arnebia guttata Bunge } \\
\text { (Arn-gut) } \\
\text { Boraginaceae } \\
\text { SMH-161 }\end{array}$ & $\begin{array}{l}\text { Deemok/ } \\
\text { Arnebia }\end{array}$ & Herb & Roots & $\begin{array}{l}\text { Roots are shade-dried } \\
\text { and decoction is made } \\
\text { from ground roots, which } \\
\text { is taken orally for three } \\
\text { consecutive days. }\end{array}$ & Reduce cough and cold. & $\longrightarrow$ & $\mathrm{Y}$ & $\mathrm{Y}$ & $\mathrm{Y}$ \\
\hline $\begin{array}{c}\text { Bergenia ciliata (Haw.) } \\
\text { Sternb. } \\
\text { (Ber-cil) } \\
\text { Saxifragaceae } \\
\text { SMH-202 }\end{array}$ & $\begin{array}{c}\text { Pasanbheda/ } \\
\text { Winter begonia }\end{array}$ & Herb & Whole plant & $\begin{array}{l}\text { Plant is sun-dried, } \\
\text { ground to a powder, and } \\
\text { taken with water orally. }\end{array}$ & Renal calculi. & $\begin{array}{l}\text { Roots are used for } \\
\text { making herbal tea. }\end{array}$ & $\mathrm{Y}$ & $\mathrm{Y}$ & $\mathrm{Y}$ \\
\hline $\begin{array}{c}\text { Berberis dictyophylla } \\
\text { Franch. } \\
\text { (Ber-dic) } \\
\text { Berberidaceae } \\
\text { SMH-124 } \\
\end{array}$ & $\begin{array}{c}\text { Sker-pa/ } \\
\text { Netleaf barberry }\end{array}$ & Shrub & Bark & $\begin{array}{l}\text { Tea is obtained by boiling } \\
\text { the bark in water for } \\
\text { more than } 40 \text { min, and } \\
\text { taken orally. }\end{array}$ & $\begin{array}{l}\text { Diabetes, trachoma, } \\
\text { and nephritis. }\end{array}$ & - & $\mathrm{N}$ & $\mathrm{Y}$ & $\mathrm{N}$ \\
\hline $\begin{array}{c}\text { Berberis ulicina Hook.f. } \\
\text { \& Thomson. } \\
\text { (Ber-uli) } \\
\text { Berberidaceae } \\
\text { SMH-184 }\end{array}$ & $\begin{array}{c}\text { Khizer, } \\
\text { Kiraring/ } \\
\text { Gorse Barberry }\end{array}$ & Shrub & $\begin{array}{l}\text { Roots } \\
\text { Bark }\end{array}$ & $\begin{array}{l}\text { Roots are taken raw, } \\
\text { dried and ground to a } \\
\text { powder, and used orally } \\
\text { with milk. }\end{array}$ & $\begin{array}{l}\text { Both the roots and the } \\
\text { bark are used for } \\
\text { body strength. }\end{array}$ & $\longrightarrow$ & $\mathrm{N}$ & $\mathrm{Y}$ & $\mathrm{N}$ \\
\hline $\begin{array}{l}\text { Carum carvi L. } \\
\text { (Car-car) } \\
\text { Apiaceae } \\
\text { SMH-104 }\end{array}$ & $\begin{array}{l}\text { Kos-nyod, } \\
\text { Ajaji, } \\
\text { Nilisi shi/ } \\
\text { Caraway }\end{array}$ & Herb & Seeds & $\begin{array}{l}\text { Seeds are dried and } \\
\text { ground to a fine powder; } \\
\text { also used in natural form. }\end{array}$ & - & $\begin{array}{l}\text { Seeds are used to } \\
\text { add flavor to } \\
\text { local dishes. }\end{array}$ & $\mathrm{Y}$ & $\mathrm{Y}$ & $\mathrm{N}$ \\
\hline
\end{tabular}


Table 2. Cont

\begin{tabular}{|c|c|c|c|c|c|c|c|c|c|}
\hline \multirow{2}{*}{$\begin{array}{c}\text { Botanical Name } \\
\text { Abbreviation } \\
\text { Family } \\
\text { Voucher No. }\end{array}$} & \multirow{2}{*}{$\begin{array}{l}\text { Local Name } \\
\text { English Name }\end{array}$} & \multirow{2}{*}{ Life Form } & \multirow{2}{*}{ Parts Used } & \multirow{2}{*}{$\begin{array}{l}\text { Preparation and } \\
\text { Application }\end{array}$} & \multirow{2}{*}{ Diseases Cured } & \multirow{2}{*}{$\begin{array}{l}\text { Other Economic } \\
\text { Applications }\end{array}$} & \multicolumn{3}{|c|}{ Ethnic Groups } \\
\hline & & & & & & & Balti & Brokpa & Beda \\
\hline $\begin{array}{l}\text { Cortia depressa (D. } \\
\text { Don) C. Norman. } \\
\text { (Cor-dep) } \\
\text { Apiaceae } \\
\text { SMH-158 }\end{array}$ & $\begin{array}{l}\text { Bam-po-mo-rig/ } \\
\text { Iranian knapweed }\end{array}$ & Herb & $\begin{array}{l}\text { Bulbs } \\
\text { Leaves }\end{array}$ & $\begin{array}{l}\text { Bulbs are made into paste } \\
\text { and applied topically. } \\
\text { Decoction is made from } \\
\text { the leaves and } \\
\text { taken orally. }\end{array}$ & $\begin{array}{l}\text { Bulbs reduce swelling } \\
\text { in limbs. } \\
\text { Leaves are used for } \\
\text { muscular spasms. }\end{array}$ & & $\mathrm{Y}$ & $\mathrm{Y}$ & $\mathrm{N}$ \\
\hline $\begin{array}{c}\text { Carthamus lanatus L. } \\
\text { (Car-lan) } \\
\text { Asteraceae } \\
\text { SMH-123 }\end{array}$ & $\begin{array}{l}\text { Gur-gum/ } \\
\text { Safflower }\end{array}$ & Herb & Flowers & $\begin{array}{c}\text { Dried flowers are ground } \\
\text { to a powder and taken } \\
\text { orally with } \\
\text { lukewarm water. }\end{array}$ & $\begin{array}{l}\text { Liver disorders, hepatitis, } \\
\text { anemia, fever, purify blood, } \\
\text { and bleeding. }\end{array}$ & & $\mathrm{Y}$ & Y & $\mathrm{Y}$ \\
\hline $\begin{array}{c}\text { Cosmos bipinnatus Cav. } \\
\text { (Cos-bip) } \\
\text { Asteraceae } \\
\text { SMH-121 }\end{array}$ & $\begin{array}{l}\text { Pun-da-Re-ka, } \\
\text { Krang tha ri/ } \\
\text { Garden cosmos }\end{array}$ & Herb & Roots & $\begin{array}{l}\text { Roots are dried and } \\
\text { ground, then mixed with } \\
\text { water, taken orally. }\end{array}$ & $\begin{array}{l}\text { Stops bleeding and } \\
\text { blood fever. }\end{array}$ & & Y & Y & $\mathrm{N}$ \\
\hline $\begin{array}{l}\text { Codonopsis ovata } \\
\text { Benth. } \\
\text { (Cod-ova) } \\
\text { Campanulaceae } \\
\text { SMH-181 }\end{array}$ & $\begin{array}{l}\text { Klup-dud-rdo- } \\
\text { rje/ } \\
\text { Kashmir bonnet } \\
\text { bellflower }\end{array}$ & Herb & $\begin{array}{l}\text { Leaves } \\
\text { Flowers }\end{array}$ & $\begin{array}{l}\text { Poultice is made from } \\
\text { leaves and used topically. } \\
\text { Flowers are dried and } \\
\text { ground to a powder, } \\
\text { mixed with water and } \\
\text { made into paste, and } \\
\text { applied topically. }\end{array}$ & $\begin{array}{l}\text { Leaves are used against } \\
\text { arthritis, rheumatism, and } \\
\text { elephantiasis. } \\
\text { Flowers are used to treat } \\
\text { leprosy, nerve disorders, } \\
\text { stiffening of ligaments and } \\
\text { tendons, planetary diseases, } \\
\text { and diseases caused by } \\
\text { evil spirits. }\end{array}$ & - & $\mathrm{Y}$ & Y & $\mathrm{N}$ \\
\hline
\end{tabular}


Table 2. Cont.

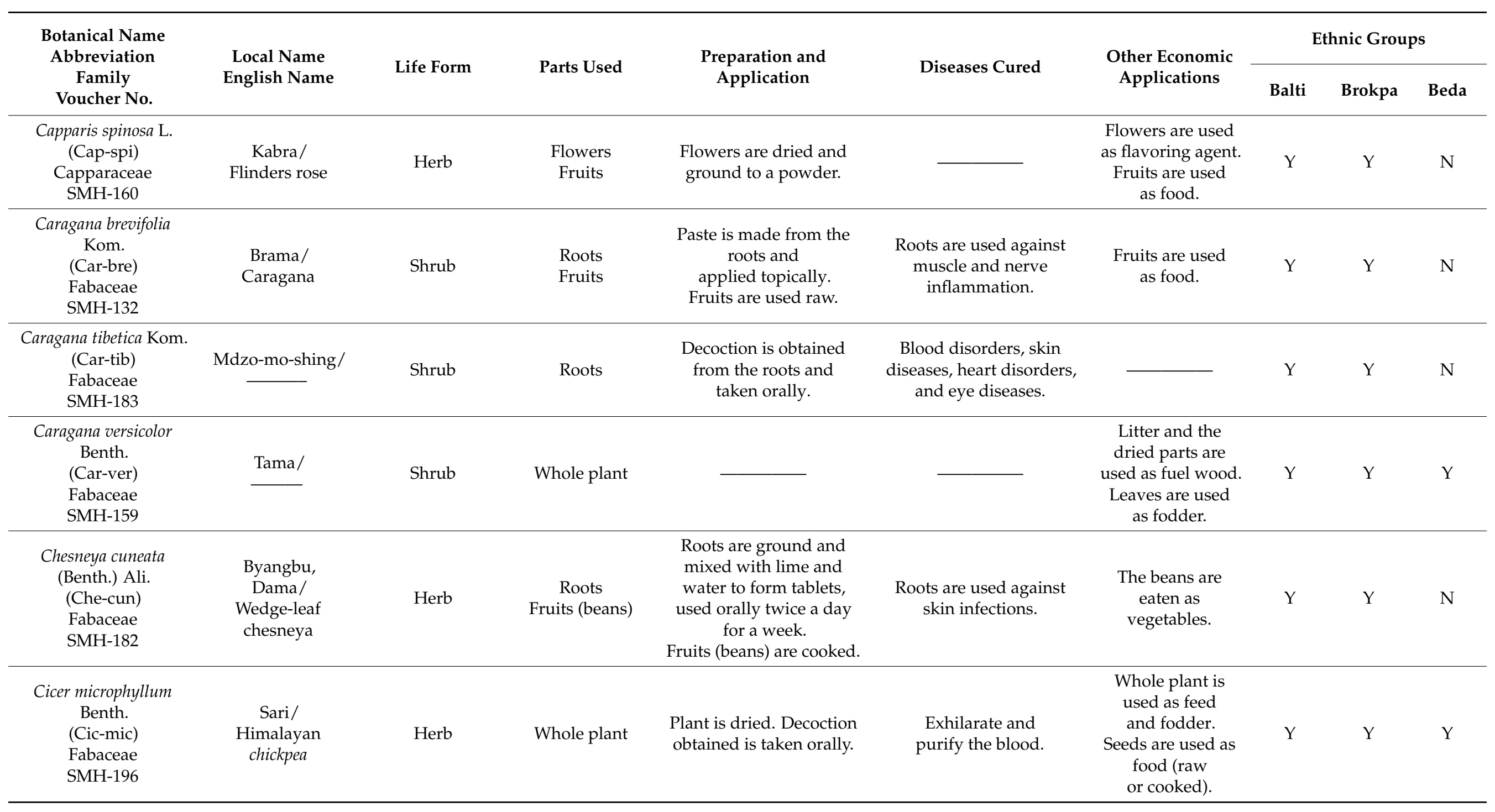


Table 2. Cont

\begin{tabular}{|c|c|c|c|c|c|c|c|c|c|}
\hline \multirow{2}{*}{$\begin{array}{c}\text { Botanical Name } \\
\text { Abbreviation } \\
\text { Family } \\
\text { Voucher No. }\end{array}$} & \multirow{2}{*}{$\begin{array}{l}\text { Local Name } \\
\text { English Name }\end{array}$} & \multirow{2}{*}{ Life Form } & \multirow{2}{*}{ Parts Used } & \multirow{2}{*}{$\begin{array}{l}\text { Preparation and } \\
\text { Application }\end{array}$} & \multirow{2}{*}{ Diseases Cured } & \multirow{2}{*}{$\begin{array}{l}\text { Other Economic } \\
\text { Applications }\end{array}$} & \multicolumn{3}{|c|}{ Ethnic Groups } \\
\hline & & & & & & & Balti & Brokpa & Beda \\
\hline $\begin{array}{c}\text { Corydalis govaniana } \\
\text { Wall. } \\
\text { (Cor-gov) } \\
\text { Papaveraceae } \\
\text { SMH-134 }\end{array}$ & Stong-zil/ & Herb & $\begin{array}{l}\text { Roots } \\
\text { Leaves }\end{array}$ & $\begin{array}{l}\text { Small maize-like pellets } \\
\text { are made from the roots } \\
\text { by grinding and } \\
\text { mixing water. } \\
\text { Decoction is made from } \\
\text { the leaves and } \\
\text { taken orally. }\end{array}$ & $\begin{array}{l}\text { Roots are used as } \\
\text { antipyretic and diuretic. } \\
\text { Leaves are used against } \\
\text { gastric pain, muscular pain, } \\
\text { contagious fever, eye } \\
\text { diseases, and swellings. }\end{array}$ & & $\mathrm{N}$ & Y & $\mathrm{N}$ \\
\hline $\begin{array}{c}\text { Clematis montana } \\
\text { Buch. -Ham. ex DC. } \\
\text { (Cle-mon) } \\
\text { Ranunculaceae } \\
\text { SMH-133 } \\
\end{array}$ & $\begin{array}{l}\text { Dbye-mong- } \\
\text { karpo/ } \\
\text { Himalayan } \\
\text { clematis }\end{array}$ & Herb & $\begin{array}{l}\text { Stem } \\
\text { Flowers }\end{array}$ & $\begin{array}{l}\text { Stem and flowers are } \\
\text { dried in the sun and } \\
\text { ground to a powder, } \\
\text { which is used with water } \\
\text { in the morning. }\end{array}$ & $\begin{array}{l}\text { Aerial parts (stem and } \\
\text { flowers) are used to } \\
\text { treat diabetes. }\end{array}$ & $\longrightarrow$ & $\mathrm{N}$ & Y & $\mathrm{N}$ \\
\hline $\begin{array}{l}\text { Clematis tibetana } \\
\text { Kuntze. } \\
\text { (Cle-tib) } \\
\text { Ranunculaceae } \\
\text { SMH-122 }\end{array}$ & $\begin{array}{c}\text { Dbi-mong, } \\
\text { Sbi-cho/ } \\
\text { Chinese clematis }\end{array}$ & Herb & $\begin{array}{l}\text { Flowers } \\
\text { Stem }\end{array}$ & $\begin{array}{c}\text { Decoction used orally } \\
\text { and paste used topically } \\
\text { are made from both stem } \\
\text { and flowers. }\end{array}$ & $\begin{array}{l}\text { Flowers are used in wind- } \\
\text { or cold-related problems, } \\
\text { tumors, wounds, } \\
\text { and arthritis. } \\
\text { Stem is used against } \\
\text { pulmonary diseases, } \\
\text { digestive heat, burns, and } \\
\text { lack of appetite. }\end{array}$ & & $\mathrm{N}$ & $\mathrm{Y}$ & $\mathrm{N}$ \\
\hline $\begin{array}{l}\text { Datura stramonium L. } \\
\text { (Dat-str) } \\
\text { Solanaceae } \\
\text { SMH-205 }\end{array}$ & $\begin{array}{l}\text { Esman/ } \\
\text { Thorn apple, } \\
\text { jimsonweed }\end{array}$ & Herb & Seeds & $\begin{array}{c}\text { Seeds are dried and } \\
\text { ground to a powder and } \\
\text { taken orally with water. } \\
\text { Dried seeds are burned to } \\
\text { produce smoke. }\end{array}$ & $\begin{array}{l}\text { Seeds are used against } \\
\text { asthma and diarrhea, and } \\
\text { as anti-inflammatory } \\
\text { medicine. Smoke is used to } \\
\text { exorcise evil spirits. }\end{array}$ & $\longrightarrow$ & Y & $\mathrm{N}$ & $\mathrm{N}$ \\
\hline $\begin{array}{c}\text { Debregeasia edulis } \\
\text { (Siebold \& Zucc.) } \\
\text { Wedd. } \\
\text { (Deb-edu) } \\
\text { Urticaceae } \\
\text { SMH-197 }\end{array}$ & Ga-dur/ & Shrub & Fruits & $\begin{array}{l}\text { Fresh and dried fruits } \\
\text { are used. }\end{array}$ & - & $\begin{array}{l}\text { Fruits are used } \\
\text { as food. }\end{array}$ & $\mathrm{N}$ & Y & $\mathrm{Y}$ \\
\hline
\end{tabular}


Table 2. Cont.

\begin{tabular}{|c|c|c|c|c|c|c|c|c|c|}
\hline \multirow{2}{*}{$\begin{array}{l}\text { Botanical Name } \\
\text { Abbreviation } \\
\text { Family } \\
\text { Voucher No. }\end{array}$} & \multirow{2}{*}{$\begin{array}{l}\text { Local Name } \\
\text { English Name }\end{array}$} & \multirow{2}{*}{ Life Form } & \multirow{2}{*}{ Parts Used } & \multirow{2}{*}{$\begin{array}{l}\text { Preparation and } \\
\text { Application }\end{array}$} & \multirow{2}{*}{ Diseases Cured } & \multirow{2}{*}{$\begin{array}{c}\text { Other Economic } \\
\text { Applications }\end{array}$} & \multicolumn{3}{|c|}{ Ethnic Groups } \\
\hline & & & & & & & Balti & Brokpa & Beda \\
\hline $\begin{array}{l}\text { Delphinium } \\
\text { brunonianum } \\
\text { Royle. } \\
\text { (Del-bru) } \\
\text { Ranunculaceae } \\
\text { SMH-105 } \\
\end{array}$ & $\begin{array}{l}\text { Bya-rgod-spos/ } \\
\text { Musk larkspur }\end{array}$ & Herb & $\begin{array}{c}\text { Stem } \\
\text { Leaves }\end{array}$ & $\begin{array}{l}\text { Paste is made from the } \\
\text { leaves and used topically. } \\
\text { Decoction is obtained } \\
\text { from the leaves and } \\
\text { taken orally. }\end{array}$ & $\begin{array}{l}\text { Stem is used to treat } \\
\text { skin diseases. } \\
\text { Leaves are used against evil } \\
\text { spirits, poisoning, epidemic } \\
\text { fever, itching, cold, cough, } \\
\text { and snakebite. }\end{array}$ & $\longrightarrow$ & $\mathrm{N}$ & $\mathrm{Y}$ & $\mathrm{Y}$ \\
\hline $\begin{array}{l}\text { Delphinium } \\
\text { cashmerianum } \\
\text { Royle. } \\
\text { (Del-cas) } \\
\text { Ranunculaceae } \\
\text { SMH-180 }\end{array}$ & $\begin{array}{c}\text { Ba-ru-ra, } \\
\text { Ladar/ } \\
\text { Kashmir larkspur }\end{array}$ & Herb & Leaves & $\begin{array}{l}\text { Fresh leaves are used } \\
\text { as such. }\end{array}$ & Insecticide. & $\longrightarrow$ & $\mathrm{N}$ & $\mathrm{Y}$ & $\mathrm{Y}$ \\
\hline $\begin{array}{l}\text { Dactylorhiza hatagirea } \\
\text { (D.Don) Soó. } \\
\text { (Dac-hat) } \\
\text { Orchidaceae } \\
\text { SMH-201 }\end{array}$ & $\begin{array}{c}\text { Ambo-lakpa/ } \\
\text { Himalayan marsh } \\
\text { orchid }\end{array}$ & Herb & Roots & $\begin{array}{c}\text { Roots are made into } \\
\text { decoction and used orally. }\end{array}$ & $\begin{array}{l}\text { Energy boosters; improve } \\
\text { health; recommended for } \\
\text { weak people. }\end{array}$ & - & $\mathrm{Y}$ & $\mathrm{Y}$ & $\mathrm{N}$ \\
\hline $\begin{array}{l}\text { Dracocephalum } \\
\text { heterophyllum } \\
\text { Benth. } \\
\text { (Dra-het) } \\
\text { Lamiaceae } \\
\text { SMH-179 }\end{array}$ & $\begin{array}{l}\text { Jib-rtse-Karpo, } \\
\text { Jib pa dkar thog,x } \\
\text { Mdzo mo nu nu/ } \\
\text { White dragonhead }\end{array}$ & Herb & $\begin{array}{l}\text { Leaves } \\
\text { Flowers }\end{array}$ & $\begin{array}{l}\text { Raw leaves are chewed. } \\
\text { Boiled in hot water to } \\
\text { get vapors. }\end{array}$ & $\begin{array}{l}\text { Leaves are used against } \\
\text { toothache and eye diseases. } \\
\text { Flowers are used to treat } \\
\text { irritation, burning } \\
\text { sensation, and pain. }\end{array}$ & $\longrightarrow$ & $\mathrm{N}$ & $\mathrm{Y}$ & $\mathrm{N}$ \\
\hline $\begin{array}{l}\text { Dracocephalum } \\
\text { tanguticum } \\
\text { Maxim. } \\
\text { (Dra-tan) } \\
\text { Lamiaceae } \\
\text { SMH-200 }\end{array}$ & $\begin{array}{c}\text { Pri-yang-ku, } \\
\text { Klum o gur gum, } \\
\text { Btsun mo gur gum/ }\end{array}$ & Herb & Leaves & $\begin{array}{l}\text { Leaves are boiled in } \\
\text { water and eaten. }\end{array}$ & $\begin{array}{l}\text { Hepatitis, gastritis, } \\
\text { dizziness, arthritis, and } \\
\text { stomach ulcer. }\end{array}$ & 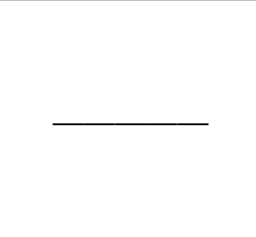 & $\mathrm{N}$ & $\mathrm{Y}$ & $\mathrm{N}$ \\
\hline
\end{tabular}


Table 2. Cont.

\begin{tabular}{|c|c|c|c|c|c|c|c|c|c|}
\hline \multirow{2}{*}{$\begin{array}{l}\text { Botanical Name } \\
\text { Abbreviation } \\
\text { Family } \\
\text { Voucher No. }\end{array}$} & \multirow{2}{*}{$\begin{array}{l}\text { Local Name } \\
\text { English Name }\end{array}$} & \multirow{2}{*}{ Life Form } & \multirow{2}{*}{ Parts Used } & \multirow{2}{*}{$\begin{array}{l}\text { Preparation and } \\
\text { Application }\end{array}$} & \multirow{2}{*}{ Diseases Cured } & \multirow{2}{*}{$\begin{array}{l}\text { Other Economic } \\
\text { Applications }\end{array}$} & \multicolumn{3}{|c|}{ Ethnic Groups } \\
\hline & & & & & & & Balti & Brokpa & Beda \\
\hline $\begin{array}{l}\text { Doronicum falconeri } \\
\text { C.B. Clarke ex Hook.f. } \\
\text { (Dor-fal) } \\
\text { Asteraceae } \\
\text { SMH-120 }\end{array}$ & $\begin{array}{c}\text { Mentok-serpo/ } \\
\text { False leopard's bane }\end{array}$ & Herb & $\begin{array}{l}\text { Leaves } \\
\text { Flowers }\end{array}$ & $\begin{array}{l}\text { Paste is made from both } \\
\text { leaves and flowers. }\end{array}$ & $\begin{array}{l}\text { Leaves are used to treat } \\
\text { diphtheria and viral and } \\
\text { bacterial diseases. } \\
\text { Flowers are used to treat } \\
\text { swelling and pain due to } \\
\text { hypertension. }\end{array}$ & & $\mathrm{N}$ & $\mathrm{Y}$ & $\mathrm{N}$ \\
\hline $\begin{array}{c}\text { Echinops cornigerus } \\
\text { DC. } \\
\text { (Ech-cor) } \\
\text { Asteraceae } \\
\text { SMH-135 }\end{array}$ & $\begin{array}{c}\text { Aczema/ } \\
\text { Blue globe thistle }\end{array}$ & Herb & $\begin{array}{l}\text { Roots } \\
\text { Leave }\end{array}$ & $\begin{array}{l}\text { Roots are powdered and } \\
\text { mixed with curd and } \\
\text { used topically. } \\
\text { Leaves are dried and } \\
\text { ground to form small } \\
\text { tablets and taken with } \\
\text { water orally. }\end{array}$ & $\begin{array}{l}\text { Roots are used to treat } \\
\text { yellowish coloration of eyes } \\
\text { due to jaundice. } \\
\text { Leaves are used to treat } \\
\text { septic wounds. }\end{array}$ & - & Y & Y & $\mathrm{N}$ \\
\hline $\begin{array}{l}\text { Ephedra gerardiana } \\
\text { Wall. ex Stapf. } \\
\text { (Eph-ger) } \\
\text { Ephedraceae } \\
\text { SMH-119 }\end{array}$ & $\begin{array}{l}\text { Tsepat, Chhepat/ } \\
\text { Gerard jointfir }\end{array}$ & Herb & $\begin{array}{l}\text { Leaves } \\
\text { Stem } \\
\text { Flowers }\end{array}$ & $\begin{array}{l}\text { Leaves are made into } \\
\text { paste and } \\
\text { applied topically. } \\
\text { Young stem twigs are cut } \\
\text { into the size of a } \\
\text { toothbrush. } \\
\text { Flowers are made into } \\
\text { decoction, used orally. }\end{array}$ & $\begin{array}{l}\text { Leaves are used to } \\
\text { reduce fever. } \\
\text { Flowers are used to treat } \\
\text { hepatic diseases, rheumatism, } \\
\text { and bronchial asthma. }\end{array}$ & $\begin{array}{c}\text { Stem is an } \\
\text { alternative to } \\
\text { toothbrush and paste. } \\
\text { Dried stem is also } \\
\text { used as fuel. }\end{array}$ & $\mathrm{N}$ & $\mathrm{Y}$ & $\mathrm{N}$ \\
\hline $\begin{array}{c}\text { Epilobium latifolium L. } \\
\text { (Epi-lat) } \\
\text { Onagraceae } \\
\text { SMH-106 }\end{array}$ & $\begin{array}{l}\text { Byar-pan-chu-tse/ } \\
\text { Dwarf fireweed }\end{array}$ & Herb & $\begin{array}{l}\text { Flowers } \\
\text { Leaves }\end{array}$ & $\begin{array}{l}\text { Flowers are made into } \\
\text { paste and used topically. } \\
\text { Decoction is made from } \\
\text { the leaves and } \\
\text { used orally. }\end{array}$ & $\begin{array}{l}\text { Flowers are used against } \\
\text { dropsy. } \\
\text { Leaves are used against urine } \\
\text { obstruction, severe pain, fever } \\
\text { due to elephantiasis, arthritis, } \\
\text { and pimples. }\end{array}$ & & Y & $\mathrm{Y}$ & $\mathrm{N}$ \\
\hline $\begin{array}{c}\text { Fritillaria verticillata } \\
\text { Willd. } \\
\text { (Fri-ver) } \\
\text { Liliaceae } \\
\text { SMH-189 }\end{array}$ & Shri khanta/ & Herb & Bulbs & $\begin{array}{l}\text { Bulbs are ground and } \\
\text { mixed with honey and } \\
\text { taken orally. }\end{array}$ & $\begin{array}{l}\text { Antidote, antitussive, } \\
\text { astringent, expectorant, } \\
\text { and purgative. }\end{array}$ & 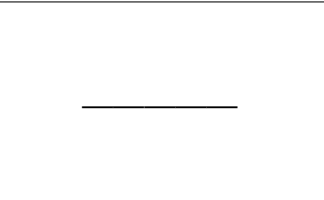 & Y & Y & Y \\
\hline
\end{tabular}


Table 2. Cont

\begin{tabular}{|c|c|c|c|c|c|c|c|c|c|}
\hline \multirow{2}{*}{$\begin{array}{c}\text { Botanical Name } \\
\text { Abbreviation } \\
\text { Family } \\
\text { Voucher No. }\end{array}$} & \multirow{2}{*}{$\begin{array}{l}\text { Local Name } \\
\text { English Name }\end{array}$} & \multirow{2}{*}{ Life Form } & \multirow{2}{*}{ Parts Used } & \multirow{2}{*}{$\begin{array}{l}\text { Preparation and } \\
\text { Application }\end{array}$} & \multirow{2}{*}{ Diseases Cured } & \multirow{2}{*}{$\begin{array}{l}\text { Other Economic } \\
\text { Applications }\end{array}$} & \multicolumn{3}{|c|}{ Ethnic Groups } \\
\hline & & & & & & & Balti & Brokpa & Beda \\
\hline $\begin{array}{c}\text { Ferula jaeschkeana } \\
\text { Vatke. } \\
\text { (Fer-jae) } \\
\text { Apiaceae } \\
\text { SMH-178 }\end{array}$ & $\begin{array}{c}\text { Wild Hing, } \\
\text { Tru-nag/ } \\
\text { Wild Asafoetida }\end{array}$ & Herb & Roots & $\begin{array}{l}\text { Roots are dried and } \\
\text { ground to a powder, } \\
\text { mixed with spring water } \\
\text { to form a paste, and } \\
\text { used topically. }\end{array}$ & Septic wounds and cuts. & - & $\mathrm{Y}$ & $\mathrm{Y}$ & $\mathrm{Y}$ \\
\hline $\begin{array}{l}\text { Gentiana chirayita } \\
\text { Roxb. ex Flem. } \\
\text { (Gen-chi) } \\
\text { Gentianaceae } \\
\text { SMH-118 }\end{array}$ & $\begin{array}{c}\text { Tik-ta/ } \\
\text { Indian gentian }\end{array}$ & Herb & Whole plant & $\begin{array}{l}\text { Decoction is made and } \\
\text { taken orally. }\end{array}$ & $\begin{array}{l}\text { Febrifuge, carminative, } \\
\text { expectorant, laxative, } \\
\text { stomachic, anthelmintic, } \\
\text { and anti-diarrheal. }\end{array}$ & & $\mathrm{N}$ & Y & $\mathrm{N}$ \\
\hline $\begin{array}{c}\text { Gentiana urnula Harry } \\
\text { Sm. } \\
\text { (Gen-urn) } \\
\text { Gentianaceae } \\
\text { SMH-177 }\end{array}$ & $\begin{array}{c}\text { Ganga-chung, } \\
\text { Rgyal mtshan ganga } \\
\text { chung, } \\
\text { Gangs las skyes/ } \\
\text { Starfish succulent }\end{array}$ & Herb & Whole plant & $\begin{array}{l}\text { Decoction is made and } \\
\text { taken orally. }\end{array}$ & $\begin{array}{l}\text { Prominent laxative, } \\
\text { anthelmintic, and } \\
\text { anti-diarrheal. }\end{array}$ & - & $\mathrm{N}$ & Y & $\mathrm{N}$ \\
\hline $\begin{array}{l}\text { Gentiana algida Pall. } \\
\text { (Gen-alg) } \\
\text { Gentianaceae } \\
\text { SMH-157 }\end{array}$ & $\begin{array}{c}\text { Tiktas, } \\
\text { Spang-gyan-karpo, } \\
\text { Ta pa ni/ } \\
\text { whitish gentian }\end{array}$ & Herb & Flowers & $\begin{array}{l}\text { Flowers are dried, } \\
\text { ground, mixed with lime } \\
\text { and honey, and } \\
\text { taken orally. }\end{array}$ & $\begin{array}{l}\text { To reduce the inflammation } \\
\text { of the pharynx, bronchitis, } \\
\text { cough, hoarseness of throat, } \\
\text { excess sputum, and toxic } \\
\text { and epidemic fevers. }\end{array}$ & - & $\mathrm{N}$ & Y & $\mathrm{N}$ \\
\hline $\begin{array}{l}\text { Herminium monorchis } \\
\text { (L.) R.Br. } \\
\text { (Her-mon) } \\
\text { Orchidaceae } \\
\text { SMH-198 }\end{array}$ & $\begin{array}{l}\text { Bye-lche-lag-pa/ } \\
\text { Musk orchid }\end{array}$ & Herb & Roots & $\begin{array}{l}\text { Fresh and dry roots are } \\
\text { half ground and boiled in } \\
\text { water, and taken orally. }\end{array}$ & $\begin{array}{l}\text { Rejuvenates health, } \\
\text { increases sperm count; vital } \\
\text { essence; acts as aphrodisiac } \\
\text { and improves kidney heat. }\end{array}$ & & $\mathrm{N}$ & Y & $\mathrm{N}$ \\
\hline $\begin{array}{c}\text { Hippophae rhamnoides } \\
\text { L. } \\
\text { (Hip-rha) } \\
\text { Elaeagnaceae } \\
\text { SMH-156 }\end{array}$ & $\begin{array}{l}\text { Gla ba tsher ma, } \\
\text { Gle tsher, } \\
\text { Star ru/ } \\
\text { Sea-buckthorn }\end{array}$ & Herb & Whole plant & $\begin{array}{l}\text { Plant is dried and } \\
\text { powdered. Decoction is } \\
\text { made and taken orally. }\end{array}$ & $\begin{array}{l}\text { Anti-aging, anti-cold, } \\
\text { memory sharpener, and } \\
\text { energy booster. }\end{array}$ & $\begin{array}{l}\text { Whole plant is } \\
\text { used as fuel } \\
\text { and fodder. }\end{array}$ & Y & Y & $\mathrm{Y}$ \\
\hline
\end{tabular}


Table 2. Cont.

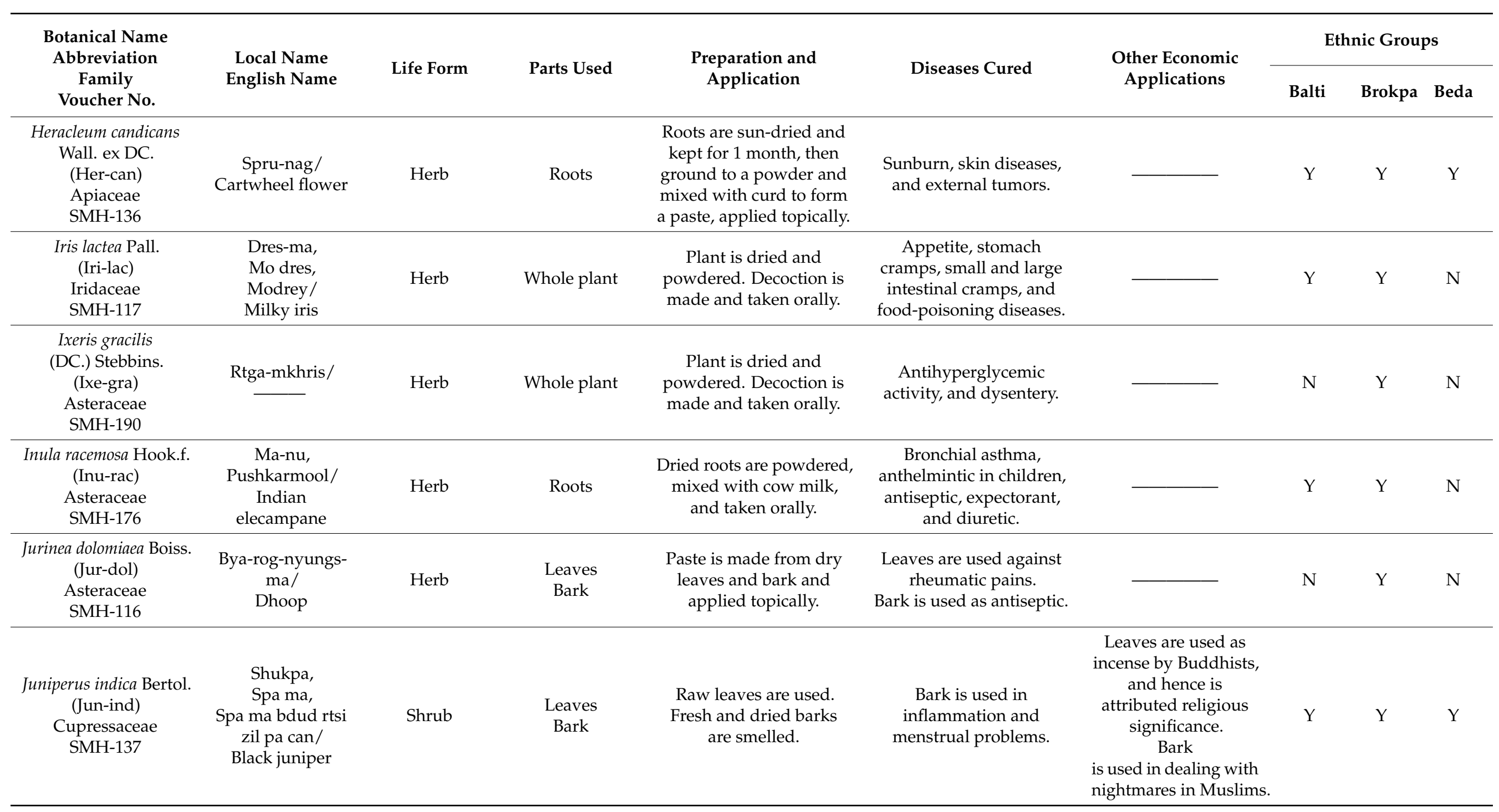


Table 2. Cont.

\begin{tabular}{|c|c|c|c|c|c|c|c|c|c|}
\hline \multirow{2}{*}{$\begin{array}{l}\text { Botanical Name } \\
\text { Abbreviation } \\
\text { Family } \\
\text { Voucher No. }\end{array}$} & \multirow{2}{*}{$\begin{array}{l}\text { Local Name } \\
\text { English Name }\end{array}$} & \multirow{2}{*}{ Life Form } & \multirow{2}{*}{ Parts Used } & \multirow{2}{*}{$\begin{array}{l}\text { Preparation and } \\
\text { Application }\end{array}$} & \multirow{2}{*}{ Diseases Cured } & \multirow{2}{*}{$\begin{array}{l}\text { Other Economic } \\
\text { Applications }\end{array}$} & \multicolumn{3}{|c|}{ Ethnic Groups } \\
\hline & & & & & & & Balti & Brokpa & Beda \\
\hline $\begin{array}{l}\text { Juniperus recurva Buch. } \\
\text {-Ham. ex D.Don. } \\
\text { (Jun-rec) } \\
\text { Cupressaceae } \\
\text { SMH-155 }\end{array}$ & $\begin{array}{l}\text { Shug-pa-tser-chan/ } \\
\text { Drooping juniper }\end{array}$ & Shrub & $\begin{array}{l}\text { Leaves } \\
\text { Fruits }\end{array}$ & $\begin{array}{l}\text { Powdered leaves are used } \\
\text { with stream water and } \\
\text { taken orally. } \\
\text { Fruits are taken raw. }\end{array}$ & $\begin{array}{l}\text { Leaves are used to treat } \\
\text { kidney problems and } \\
\text { muscular spasms. } \\
\text { Fruit improves health. }\end{array}$ & & $\mathrm{Y}$ & $\mathrm{Y}$ & $\mathrm{Y}$ \\
\hline $\begin{array}{l}\text { Lactuca dissecta } \\
\text { D. Don. } \\
\text { (Lac-dis) } \\
\text { Asteraceae } \\
\text { SMH-107 }\end{array}$ & $\begin{array}{c}\text { Khala/ } \\
\text { Split-leaf lettuce }\end{array}$ & Herb & $\begin{array}{l}\text { Leaves } \\
\text { Stem }\end{array}$ & $\begin{array}{l}\text { Dried leaves and stem are } \\
\text { ground, mixed with water to } \\
\text { form paste, and } \\
\text { applied topically. }\end{array}$ & $\begin{array}{l}\text { Infections of female } \\
\text { external genital organs. }\end{array}$ & & $\mathrm{N}$ & $\mathrm{Y}$ & $\mathrm{Y}$ \\
\hline $\begin{array}{l}\text { Lonicera spinosa (Decne.) } \\
\text { Jacq. ex Walp. } \\
\text { (Lon-spi) } \\
\text { Caprifoliaceae } \\
\text { SMH-138 }\end{array}$ & $\begin{array}{c}\text { Phang-ma/ } \\
\text { Spiny Honeysuckle }\end{array}$ & Herb & Flowers & $\begin{array}{l}\text { Flowers are kept in an } \\
\text { air-tight jar for more than } 40 \\
\text { days for fermentation and } \\
\text { then taken orally. }\end{array}$ & $\begin{array}{l}\text { Asthma, headache, and } \\
\text { gynecological disorders. }\end{array}$ & & $\mathrm{Y}$ & $\mathrm{Y}$ & $\mathrm{Y}$ \\
\hline $\begin{array}{l}\text { Leonurus japonicus } \\
\text { Houtt. } \\
\text { Lamiaceae } \\
\text { (Leo-jap) } \\
\text { SMH-165 }\end{array}$ & $\begin{array}{l}\text { Zin-tig/ } \\
\text { Oriental } \\
\text { motherwort }\end{array}$ & Herb & $\begin{array}{l}\text { Leaves } \\
\text { Stem }\end{array}$ & $\begin{array}{l}\text { Leaves are shade-dried and } \\
\text { powdered, mixed with water, } \\
\text { and taken orally. } \\
\text { Decoction is made from the } \\
\text { stem and taken orally. }\end{array}$ & $\begin{array}{l}\text { Leaves regulate menstrual } \\
\text { disturbance, dysmenorrhea, } \\
\text { amenorrhea, blood stasis, } \\
\text { and postpartum } \\
\text { hemorrhage. } \\
\text { Stem is used as diuretic. }\end{array}$ & & $\mathrm{N}$ & $\mathrm{Y}$ & $\mathrm{N}$ \\
\hline $\begin{array}{c}\text { Lagotis cashmeriana } \\
\text { Rupr. } \\
\text { (Lag-cas) } \\
\text { Plantaginaceae } \\
\text { SMH-175 }\end{array}$ & $\begin{array}{l}\text { Hong-len/ } \\
\text { Kashmir lagotis }\end{array}$ & Herb & Roots & $\begin{array}{l}\text { Roots are shade-dried and } \\
\text { boiled in water and } \\
\text { taken orally. }\end{array}$ & $\begin{array}{l}\text { Reduce inflammation, vital } \\
\text { fever, liver fever, lungs } \\
\text { fever, intestinal fever, } \\
\text { poisonous, painful cramps, } \\
\text { muscular spasms, and } \\
\text { child dysentery. }\end{array}$ & - & $\mathrm{N}$ & $\mathrm{Y}$ & $\mathrm{N}$ \\
\hline $\begin{array}{c}\text { Myricaria squamosa Desv. } \\
\text { (Myr-squ) } \\
\text { Tamaricaceae } \\
\text { SMH-174 }\end{array}$ & $\begin{array}{c}\text { Om-bu/ } \\
\text { Scaly false tamarisk }\end{array}$ & Shrub & Stem & $\begin{array}{l}\text { Dried stem is ground and } \\
\text { decoction is made; } \\
\text { taken orally. }\end{array}$ & Food poisoning. & - & $\mathrm{Y}$ & $\mathrm{Y}$ & $\mathrm{N}$ \\
\hline
\end{tabular}


Table 2. Cont

\begin{tabular}{|c|c|c|c|c|c|c|c|c|c|}
\hline \multirow{2}{*}{$\begin{array}{l}\text { Botanical Name } \\
\text { Abbreviation } \\
\text { Family } \\
\text { Voucher No. }\end{array}$} & \multirow{2}{*}{$\begin{array}{l}\text { Local Name } \\
\text { English Name }\end{array}$} & \multirow{2}{*}{ Life Form } & \multirow{2}{*}{ Parts Used } & \multirow{2}{*}{$\begin{array}{l}\text { Preparation and } \\
\text { Application }\end{array}$} & \multirow{2}{*}{ Diseases Cured } & \multirow{2}{*}{$\begin{array}{l}\text { Other Economic } \\
\text { Applications }\end{array}$} & \multicolumn{3}{|c|}{ Ethnic Groups } \\
\hline & & & & & & & Balti & Brokpa & Beda \\
\hline $\begin{array}{l}\text { Myricaria elegans } \\
\text { Royle. } \\
\text { (Myr-ele) } \\
\text { Tamaricaceae } \\
\text { SMH-115 }\end{array}$ & $\begin{array}{l}\text { Hom-bu/ } \\
\text { Elegant false } \\
\text { tamarisk }\end{array}$ & Shrub & Stem & $\begin{array}{l}\text { Stem is ground and } \\
\text { boiled in water for } 20 \mathrm{~min} \\
\text { and then eaten. }\end{array}$ & $\begin{array}{l}\text { Fever of lungs, diarrhea, } \\
\text { arthritis, uterine bleeding, } \\
\text { stomach pain, and headache. }\end{array}$ & $\longrightarrow$ & $\mathrm{Y}$ & $\mathrm{Y}$ & $\mathrm{N}$ \\
\hline $\begin{array}{c}\text { Meconopsis aculeata } \\
\text { Royle. } \\
\text { (Mec-acu) } \\
\text { Papaveraceae } \\
\text { SMH-154 }\end{array}$ & $\begin{array}{l}\text { Ud-pal-sngon-po/ } \\
\text { Poppy }\end{array}$ & Herb & Leaves & $\begin{array}{l}\text { Fresh leaves are } \\
\text { powdered, mixed with } \\
\text { water to make a paste, } \\
\text { and applied topically. }\end{array}$ & Headache. & $\longrightarrow$ & $\mathrm{N}$ & Y & $\mathrm{Y}$ \\
\hline $\begin{array}{c}\text { Morina longifolia Wall. } \\
\text { ex DC. } \\
\text { (Mor-lon) } \\
\text { Caprifoliaceae } \\
\text { SMH-191 }\end{array}$ & $\begin{array}{c}\text { Spyang-tsher, } \\
\text { Bya tra pad, } \\
\text { Chang tsher karpo/ } \\
\text { Himalayan whorl } \\
\text { flower }\end{array}$ & Herb & $\begin{array}{l}\text { Roots } \\
\text { Young-twigs }\end{array}$ & $\begin{array}{l}\text { Decoction is made from } \\
\text { the roots and young } \\
\text { twigs and taken orally. }\end{array}$ & $\begin{array}{l}\text { Roots are used against phlegm } \\
\text { disease, cancer, and swelling. } \\
\text { Young twigs are used for } \\
\text { indigestion. }\end{array}$ & & $\mathrm{Y}$ & $\mathrm{Y}$ & $\mathrm{N}$ \\
\hline $\begin{array}{l}\text { Nepeta floccosa } \\
\text { Benth. } \\
\text { (Nep-flo) } \\
\text { Lamiaceae } \\
\text { SMH-153 }\end{array}$ & $\begin{array}{l}\text { Shangukuram/ } \\
\text { Woolly catmint }\end{array}$ & Herb & Leaves & $\begin{array}{l}\text { Dried leaves are ground } \\
\text { to a powder. }\end{array}$ & 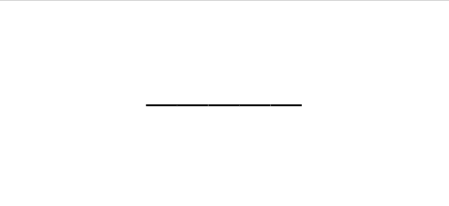 & $\begin{array}{l}\text { Leaves add flavor } \\
\text { in local dishes. }\end{array}$ & $\mathrm{Y}$ & $\mathrm{Y}$ & $\mathrm{Y}$ \\
\hline $\begin{array}{l}\text { Oxytropis microphylla } \\
\text { (Pall.) DC. } \\
\text { (Oxy-mic) } \\
\text { Fabaceae } \\
\text { SMH-139 }\end{array}$ & $\begin{array}{l}\text { Tag-sha nagpo, } \\
\text { Sngo stag sha, } \\
\text { Pa nig ho ra/ } \\
\text { Small leaved } \\
\text { locoweed }\end{array}$ & Herb & Whole plant & Fresh plant is used. & $\longrightarrow$ & $\begin{array}{l}\text { The whole plant } \\
\text { bears a strong } \\
\text { fragrance and } \\
\text { hence is kept in } \\
\text { homes. }\end{array}$ & $\mathrm{Y}$ & $\mathrm{Y}$ & $\mathrm{N}$ \\
\hline $\begin{array}{l}\text { Onosma hookeri } \\
\text { C.B. Clarke. } \\
\text { (Ono-hoo) } \\
\text { Boraginaceae } \\
\text { SMH-108 }\end{array}$ & $\begin{array}{l}\text { Bri-mog, xi hua dian } \\
\text { zi cao/ } \\
\end{array}$ & Herb & $\begin{array}{l}\text { Roots } \\
\text { Bark } \\
\text { Leaves }\end{array}$ & $\begin{array}{l}\text { Decoction is made from } \\
\text { the roots, bark, and } \\
\text { leaves and used orally. }\end{array}$ & $\begin{array}{c}\text { Roots are used against asthma, } \\
\text { cough, and fever. } \\
\text { Bark is used against } \\
\text { gonorrhea and leprosy. } \\
\text { Leaves are used against } \\
\text { phthisis. }\end{array}$ & $\longrightarrow$ & $\mathrm{Y}$ & $\mathrm{Y}$ & $\mathrm{Y}$ \\
\hline
\end{tabular}


Table 2. Cont.

\begin{tabular}{|c|c|c|c|c|c|c|c|c|c|}
\hline \multirow{2}{*}{$\begin{array}{l}\text { Botanical Name } \\
\text { Abbreviation } \\
\text { Family } \\
\text { Voucher No. }\end{array}$} & \multirow{2}{*}{$\begin{array}{l}\text { Local Name } \\
\text { English Name }\end{array}$} & \multirow{2}{*}{ Life Form } & \multirow{2}{*}{ Parts Used } & \multirow{2}{*}{$\begin{array}{l}\text { Preparation and } \\
\text { Application }\end{array}$} & \multirow{2}{*}{ Diseases Cured } & \multirow{2}{*}{$\begin{array}{l}\text { Other Economic } \\
\text { Applications }\end{array}$} & \multicolumn{3}{|c|}{ Ethnic Groups } \\
\hline & & & & & & & Balti & Brokpa & Beda \\
\hline $\begin{array}{l}\text { Picris hieracioides } \\
\text { Sibth. \& Sm. } \\
\text { (Pic-hie) } \\
\text { Asteraceae } \\
\text { SMH-152 }\end{array}$ & $\begin{array}{l}\text { Rgya-mkhur, } \\
\text { Aam dkar, } \\
\text { Gyakhur/ } \\
\text { Hawkweed } \\
\text { oxtongue }\end{array}$ & Herb & Whole plant & $\begin{array}{l}\text { Plant is dried and } \\
\text { powdered, mixed with } \\
\text { water, and taken orally. }\end{array}$ & $\begin{array}{l}\text { Gastro-intestinal disorder, } \\
\text { blood disorder, bile disorder, } \\
\text { chronic fever, and } \\
\text { contagious fever. }\end{array}$ & & $\mathrm{N}$ & $\mathrm{Y}$ & $\mathrm{Y}$ \\
\hline $\begin{array}{c}\text { Pedicularis } \\
\text { cheilanthifolia Schrenk. } \\
\text { (Ped-che) } \\
\text { Orobanchaceae } \\
\text { SMH-192 }\end{array}$ & $\begin{array}{l}\text { Lug-ru-smog-po, } \\
\text { sui mi jue ye/ } \\
\text { White Lousewort }\end{array}$ & Herb & Stem & $\begin{array}{l}\text { Stem is dried and ground to } \\
\text { a fine powder and taken } \\
\text { with water orally. }\end{array}$ & Stomach ache. & $\longrightarrow$ & Y & $\mathrm{Y}$ & $\mathrm{Y}$ \\
\hline $\begin{array}{c}\text { Pedicularis longiflora } \\
\text { Rudolph. } \\
\text { (Ped-lon) } \\
\text { Orobanchaceae } \\
\text { SMH-166 } \\
\end{array}$ & $\begin{array}{l}\text { Lug-ru-ser-po/ } \\
\text { Long tube } \\
\text { lousewort }\end{array}$ & Herb & Flowers & $\begin{array}{l}\text { Decoction is obtained from } \\
\text { dried flowers and } \\
\text { used orally. }\end{array}$ & $\begin{array}{l}\text { Diuretic, liver, and gallbladder } \\
\text { disorders, excessive seminal } \\
\text { discharge, and edema. }\end{array}$ & - & $\mathrm{Y}$ & $\mathrm{Y}$ & $\mathrm{Y}$ \\
\hline $\begin{array}{l}\text { Piper longum L. } \\
\text { (Pip-lon) } \\
\text { Piperaceae } \\
\text { SMH-173 }\end{array}$ & $\begin{array}{l}\text { Pi-pi-ling, } \\
\text { Byi ril ma, } \\
\text { Yul dbus/ } \\
\text { Long pepper }\end{array}$ & Herb & $\begin{array}{l}\text { Roots } \\
\text { Fruits }\end{array}$ & $\begin{array}{l}\text { Roots and fruits are dried } \\
\text { and boiled in water for some } \\
\text { time and then taken orally. }\end{array}$ & $\begin{array}{l}\text { Prevention and treatment of } \\
\text { prostate cancer. }\end{array}$ & & Y & $\mathrm{Y}$ & $\mathrm{Y}$ \\
\hline $\begin{array}{c}\text { Plantago depressa } \\
\text { Willd. } \\
\text { (Pla-dep) } \\
\text { Plantaginaceae } \\
\text { SMH-140 }\end{array}$ & $\begin{array}{l}\text { Tha-ram/ } \\
\text { Blond psyllium }\end{array}$ & Herb & $\begin{array}{l}\text { Leaves } \\
\text { Stem } \\
\text { Flowers }\end{array}$ & $\begin{array}{l}\text { Leaves are dried, powdered, } \\
\text { and taken with lukewarm } \\
\text { water orally. } \\
\text { Fresh stem is ground, made } \\
\text { into a paste, and } \\
\text { applied topically. } \\
\text { Decoction is obtained from } \\
\text { the flowers and used orally. }\end{array}$ & $\begin{array}{c}\text { Leaves are used } \\
\text { against dysentery. } \\
\text { Stem is used against } \\
\text { burn wounds. } \\
\text { Flowers are used against } \\
\text { bleeding and inflammation. }\end{array}$ & & $\mathrm{N}$ & $\mathrm{Y}$ & $\mathrm{Y}$ \\
\hline $\begin{array}{l}\text { Polygonum aviculare L. } \\
\text { (Pol-avi) } \\
\text { Polygonaceae } \\
\text { SMH-151 }\end{array}$ & $\begin{array}{l}\text { Byi-na-sa/ } \\
\text { Knotgrass }\end{array}$ & Herb & Whole plant & $\begin{array}{l}\text { Decoction is obtained by } \\
\text { grinding and boiling in } \\
\text { water for more than } 3 \mathrm{~h} \text { and } \\
\text { taken orally. }\end{array}$ & $\begin{array}{l}\text { Obstruction of urine, burning } \\
\text { sensation during urination, } \\
\text { jaundice, and dermatological } \\
\text { diseases with itching. }\end{array}$ & & Y & Y & Y \\
\hline
\end{tabular}


Table 2. Cont

\begin{tabular}{|c|c|c|c|c|c|c|c|c|c|}
\hline \multirow{2}{*}{$\begin{array}{l}\text { Botanical Name } \\
\text { Abbreviation } \\
\text { Family } \\
\text { Voucher No. }\end{array}$} & \multirow{2}{*}{$\begin{array}{l}\text { Local Name } \\
\text { English Name }\end{array}$} & \multirow{2}{*}{ Life Form } & \multirow{2}{*}{ Parts Used } & \multirow{2}{*}{$\begin{array}{l}\text { Preparation and } \\
\text { Application }\end{array}$} & \multirow{2}{*}{ Diseases Cured } & \multirow{2}{*}{$\begin{array}{l}\text { Other Economic } \\
\text { Applications }\end{array}$} & \multicolumn{3}{|c|}{ Ethnic Groups } \\
\hline & & & & & & & Balti & Brokpa & Beda \\
\hline $\begin{array}{l}\text { Potentilla anserina L. } \\
\text { (Pot-ans) } \\
\text { Rosaceae } \\
\text { SMH-144 }\end{array}$ & $\begin{array}{l}\text { Gro-lo sa-hdzin, } \\
\text { Gro ma, } \\
\text { Dolo sazin/ } \\
\text { Silverweed }\end{array}$ & Herb & Leaves & $\begin{array}{l}\text { Leaves are dried in } \\
\text { homes, powdered, and } \\
\text { used orally with water. }\end{array}$ & $\begin{array}{l}\text { Diarrhea/dysentery, and } \\
\text { health tonic. }\end{array}$ & & Y & Y & Y \\
\hline $\begin{array}{l}\text { Picrorhiza kurroa } \\
\text { Royle. } \\
\text { (Pic-kur) } \\
\text { Scrophulariaceae } \\
\text { SMH-199 }\end{array}$ & $\begin{array}{l}\text { Hong-len, Kutki/ } \\
\text { Katuk }\end{array}$ & Herb & $\begin{array}{l}\text { Roots } \\
\text { Flowers }\end{array}$ & $\begin{array}{l}\text { Roots are dried in shade, } \\
\text { powdered, mixed with } \\
\text { water, and taken orally. } \\
\text { Dried flowers are } \\
\text { hand-rubbed into } \\
\text { lukewarm water and } \\
\text { used orally. }\end{array}$ & $\begin{array}{l}\text { Roots are used to treat } \\
\text { asthmatic disorders } \\
\text { and fever. } \\
\text { Flowers are used in } \\
\text { blood purification. }\end{array}$ & - & $\mathrm{N}$ & $\mathrm{Y}$ & $\mathrm{N}$ \\
\hline $\begin{array}{l}\text { Physochlaina praealta } \\
\text { (Decne.) Miers. } \\
\text { (Phy-pra) } \\
\text { Solanaceae } \\
\text { SMH-109 }\end{array}$ & $\begin{array}{c}\text { Lantang, } \\
\text { Thang-phron- } \\
\text { nagpo/ } \\
\text { Praealtus,-a,-um }\end{array}$ & Herb & $\begin{array}{l}\text { Seeds } \\
\text { Leaves }\end{array}$ & $\begin{array}{l}\text { Seeds are ground to a fine } \\
\text { powder and used orally } \\
\text { with lukewarm water. } \\
\text { Fresh leaves are ground } \\
\text { to obtain the juice, which } \\
\text { is used as eye drops. }\end{array}$ & $\begin{array}{c}\text { Seeds are used } \\
\text { as vermifuge. } \\
\text { Leaves are used against } \\
\text { eye diseases. }\end{array}$ & & $\mathrm{Y}$ & $\mathrm{Y}$ & $\mathrm{N}$ \\
\hline $\begin{array}{l}\text { Rhodiola imbricata } \\
\text { Edgew. } \\
\text { (Rho-imb) } \\
\text { Crassulaceae } \\
\text { SMH-143 }\end{array}$ & $\begin{array}{l}\text { Shro lo, } \\
\text { Cho-ngo-ngo/ } \\
\text { Greek rhodon }\end{array}$ & Herb & Roots & $\begin{array}{l}\text { Roots are boiled in water, } \\
\text { mixed with lime, and } \\
\text { taken orally. }\end{array}$ & $\begin{array}{l}\text { Lung problems, cold, } \\
\text { cough, fever, loss of energy, } \\
\text { and pulmonary complaints. }\end{array}$ & $\longrightarrow$ & Y & $\mathrm{Y}$ & $\mathrm{Y}$ \\
\hline $\begin{array}{l}\text { Rhodiola sacra } \\
\text { Prain ex Raym. } \\
\text { Hamet S.H. Fu. } \\
\text { (Rho-sac) } \\
\text { Crassulaceae } \\
\text { SMH-193 }\end{array}$ & $\begin{array}{l}\text { Srolo-marpo/ } \\
\text { Arctic root }\end{array}$ & Herb & $\begin{array}{l}\text { Roots } \\
\text { Leaves }\end{array}$ & $\begin{array}{l}\text { Roots are dried and } \\
\text { ground and taken orally } \\
\text { with water. } \\
\text { Fresh young leaves } \\
\text { are used. }\end{array}$ & $\begin{array}{l}\text { Roots are used as tonic and } \\
\text { to restore memory. }\end{array}$ & $\begin{array}{l}\text { Leaves are cooked } \\
\text { as vegetables. }\end{array}$ & Y & $\mathrm{Y}$ & $\mathrm{Y}$ \\
\hline
\end{tabular}


Table 2. Cont.

\begin{tabular}{|c|c|c|c|c|c|c|c|c|c|}
\hline \multirow{2}{*}{$\begin{array}{l}\text { Botanical Name } \\
\text { Abbreviation } \\
\text { Family } \\
\text { Voucher No. }\end{array}$} & \multirow{2}{*}{$\begin{array}{l}\text { Local Name } \\
\text { English Name }\end{array}$} & \multirow{2}{*}{ Life Form } & \multirow{2}{*}{ Parts Used } & \multirow{2}{*}{$\begin{array}{l}\text { Preparation and } \\
\text { Application }\end{array}$} & \multirow{2}{*}{ Diseases Cured } & \multirow{2}{*}{$\begin{array}{l}\text { Other Economic } \\
\text { Applications }\end{array}$} & \multicolumn{3}{|c|}{ Ethnic Groups } \\
\hline & & & & & & & Balti & Brokpa & Beda \\
\hline $\begin{array}{c}\text { Rhododendron } \\
\text { anthopogon D. Don. } \\
\text { (Rho-ant) } \\
\text { Ericaceae } \\
\text { SMH-111 }\end{array}$ & $\begin{array}{l}\text { Ba-lu/da-lis / } \\
\text { Brass }\end{array}$ & Shrub & $\begin{array}{l}\text { Leaves } \\
\text { Flowers }\end{array}$ & $\begin{array}{l}\text { Leaves and flowers are } \\
\text { dried, hand-rubbed, kept } \\
\text { in hot water for some } \\
\text { time, and taken orally. }\end{array}$ & $\begin{array}{l}\text { Leaves are used to treat } \\
\text { high-altitude diseases, chest } \\
\text { pain, weakness, obstruction } \\
\text { of vocal cords, and } \\
\text { chronic bronchitis. } \\
\text { Flowers are used against } \\
\text { stiffness of limbs, and } \\
\text { phlegm disorders. }\end{array}$ & & $\mathrm{N}$ & $\mathrm{Y}$ & Y \\
\hline $\begin{array}{l}\text { Ribes orientale Desf. } \\
\text { (Rib-ori) } \\
\text { Grossulariaceae } \\
\text { SMH-141 }\end{array}$ & $\begin{array}{l}\text { Askuta, } \\
\text { Se-rgod/ } \\
\text { Oriental } \\
\text { gooseberry }\end{array}$ & Shrub & Fruits & Raw fruits are used. & $\begin{array}{l}\text { Swollen limbs, food } \\
\text { poisoning, hepatitis, fever of } \\
\text { the gallbladder, and } \\
\text { epidemic fever. }\end{array}$ & & $\mathrm{N}$ & $\mathrm{Y}$ & $\mathrm{N}$ \\
\hline $\begin{array}{c}\text { Rheum webbianum } \\
\text { Royle. } \\
\text { (Rhe-web) } \\
\text { Polygonaceae } \\
\text { SMH-150 }\end{array}$ & $\begin{array}{l}\text { Chu-rtsa/ } \\
\text { Indian rhubarb }\end{array}$ & Herb & $\begin{array}{l}\text { Root } \\
\text { Stem } \\
\text { Leaves }\end{array}$ & $\begin{array}{c}\text { Decoction is made from } \\
\text { fresh roots and } \\
\text { taken orally. } \\
\text { Fresh stem is made into a } \\
\text { paste and } \\
\text { applied topically. } \\
\text { Raw leaves are } \\
\text { taken orally. }\end{array}$ & $\begin{array}{l}\text { Roots are used against } \\
\text { indigestion and } \\
\text { abdominal diseases. } \\
\text { Stem is used to treat boils } \\
\text { and wounds. } \\
\text { Leaves are used against } \\
\text { gastritis and as laxative } \\
\text { and astringent. }\end{array}$ & - & $\mathrm{Y}$ & $\mathrm{Y}$ & $\mathrm{N}$ \\
\hline $\begin{array}{c}\text { Ranunculus adoxifolius } \\
\text { (Ran-ado) } \\
\text { Hand. -Mazz. } \\
\text { Ranunculaceae } \\
\text { SMH-110 }\end{array}$ & $\begin{array}{c}\text { Ga-tsah-am-lche- } \\
\text { tsah/ } \\
\text { Buttercup }\end{array}$ & Herb & $\begin{array}{l}\text { Leaves } \\
\text { Flowers }\end{array}$ & $\begin{array}{l}\text { Decoction is made from } \\
\text { leaves and taken orally. } \\
\text { Fresh flowers are made } \\
\text { into a paste, applied } \\
\text { topically. }\end{array}$ & $\begin{array}{l}\text { Leaves are used to normalize } \\
\text { the digestive heat. } \\
\text { Flowers are used against old } \\
\text { wounds and dropsy. }\end{array}$ & & $\mathrm{N}$ & $\mathrm{Y}$ & $\mathrm{Y}$ \\
\hline $\begin{array}{l}\text { Rosa sericea } \\
\text { Wall. ex Lindl. } \\
\text { (Ros-ser) } \\
\text { Rosaceae } \\
\text { SMH-149 }\end{array}$ & $\begin{array}{l}\text { Shayh/ } \\
\text { Silky rose }\end{array}$ & Shrub & Whole plant & $\begin{array}{l}\text { Litter and dried plant/ } \\
\text { fresh plant is used. }\end{array}$ & 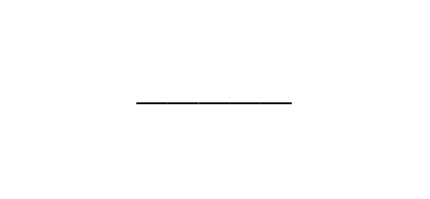 & $\begin{array}{l}\text { Whole plant is an } \\
\text { ornamental hedge. } \\
\text { Plant is also used } \\
\text { as fuel and fodder. }\end{array}$ & $\mathrm{Y}$ & $\mathrm{Y}$ & Y \\
\hline
\end{tabular}


Table 2. Cont.

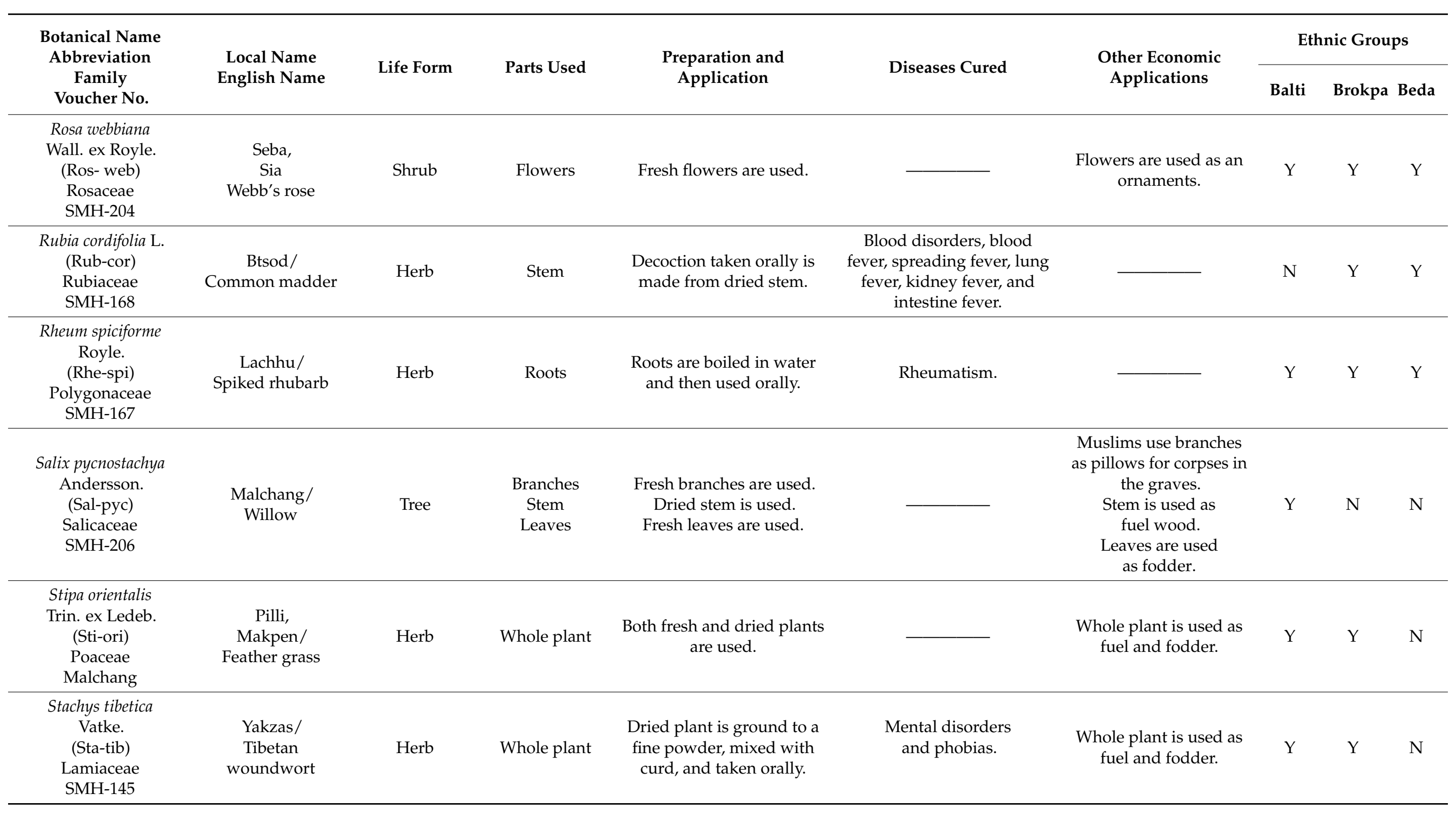


Table 2. Cont.

\begin{tabular}{|c|c|c|c|c|c|c|c|c|c|}
\hline \multirow{2}{*}{$\begin{array}{l}\text { Botanical Name } \\
\text { Abbreviation } \\
\text { Family } \\
\text { Voucher No. }\end{array}$} & \multirow{2}{*}{$\begin{array}{l}\text { Local Name } \\
\text { English Name }\end{array}$} & \multirow{2}{*}{ Life Form } & \multirow{2}{*}{ Parts Used } & \multirow{2}{*}{$\begin{array}{l}\text { Preparation and } \\
\text { Application }\end{array}$} & \multirow{2}{*}{ Diseases Cured } & \multirow{2}{*}{$\begin{array}{l}\text { Other Economic } \\
\text { Applications }\end{array}$} & \multicolumn{3}{|c|}{ Ethnic Groups } \\
\hline & & & & & & & Balti & Brokpa & Beda \\
\hline $\begin{array}{l}\text { Sedum ewersii } \\
\text { Ledeb. } \\
\text { (Sed-ewe) } \\
\text { Crassulaceae } \\
\text { SMH-169 }\end{array}$ & $\begin{array}{l}\text { Srolo-karpo/ } \\
\text { Pink sedum }\end{array}$ & Herb & Whole plant & $\begin{array}{l}\text { Fresh and dried plants } \\
\text { are used. }\end{array}$ & $\begin{array}{l}\text { Plant is given in bulk to the } \\
\text { cattle to increase } \\
\text { milk production. }\end{array}$ & $\longrightarrow$ & $\mathrm{Y}$ & $\mathrm{Y}$ & Y \\
\hline $\begin{array}{c}\text { Saussurea bracteata Decne. } \\
\text { (Sau-bra) } \\
\text { Asteraceae } \\
\text { SMH-148 }\end{array}$ & $\begin{array}{l}\text { Spang-rtsa/ } \\
\text { Narrow-leaved } \\
\text { saw-wort }\end{array}$ & Herb & Flowers & $\begin{array}{l}\text { Paste is made from flowers } \\
\text { and applied topically. }\end{array}$ & Boils. & $\begin{array}{l}\text { Flowers are used as } \\
\text { ornaments. }\end{array}$ & $\mathrm{Y}$ & $\mathrm{Y}$ & Y \\
\hline $\begin{array}{c}\text { Saussurea lappa (Decne.) } \\
\text { Sch.Bip. } \\
\text { (Sau-lap) } \\
\text { Asteraceae } \\
\text { SMH-112 } \\
\end{array}$ & $\begin{array}{l}\text { Kuth, } \\
\text { Ru-ta/ } \\
\text { Kust }\end{array}$ & Herb & Roots & $\begin{array}{l}\text { Roots are powdered and } \\
\text { taken with water orally. }\end{array}$ & Asthma and fever. & $\longrightarrow$ & $\mathrm{Y}$ & $\mathrm{Y}$ & Y \\
\hline $\begin{array}{c}\text { Tylophora fasciculata Buch. } \\
\text {-Ham. ex Wight. } \\
\text { (Tyl-fas) } \\
\text { Apocynaceae } \\
\text { SMH-194 }\end{array}$ & $\begin{array}{l}\text { Go-snyod/ } \\
\text { Country } \\
\text { ipikakyun }\end{array}$ & Herb & Fruits & Raw fruits are eaten. & $\begin{array}{l}\text { Dysentery and hot disorder } \\
\text { of gallbladder. }\end{array}$ & 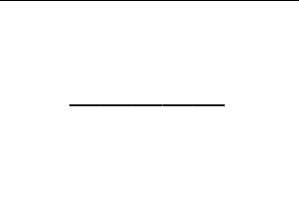 & $\mathrm{N}$ & $\mathrm{Y}$ & $\mathrm{N}$ \\
\hline $\begin{array}{l}\text { Tanacetum dolichophyllum } \\
\text { (Kitam.) Kitam. } \\
\text { (Tan-dol) } \\
\text { Asteraceae } \\
\text { SMH-113 }\end{array}$ & $\begin{array}{c}\text { Mkhan-chung-ser- } \\
\text { mgo/ } \\
\text { Garden tansy }\end{array}$ & Herb & $\begin{array}{l}\text { Leaves } \\
\text { Flowers }\end{array}$ & $\begin{array}{l}\text { Fresh leaves are ground, } \\
\text { and a little juice is obtained } \\
\text { and taken orally. } \\
\text { Flowers are boiled in water } \\
\text { for } 5 \text { min and then taken } \\
\text { orally with water. }\end{array}$ & $\begin{array}{l}\text { Leaves are used against nose } \\
\text { bleeding, swelling, and } \\
\text { inflammation of the limbs, } \\
\text { cancers, wounds, and } \\
\text { lung disorders. } \\
\text { Flowers are used against } \\
\text { renal diseases. }\end{array}$ & $\longrightarrow$ & $\mathrm{N}$ & $\mathrm{Y}$ & $\mathrm{Y}$ \\
\hline
\end{tabular}


Table 2. Cont.

\begin{tabular}{|c|c|c|c|c|c|c|c|c|c|}
\hline \multirow{2}{*}{$\begin{array}{l}\text { Botanical Name } \\
\text { Abbreviation } \\
\text { Family } \\
\text { Voucher No. }\end{array}$} & \multirow{2}{*}{$\begin{array}{l}\text { Local Name } \\
\text { English Name }\end{array}$} & \multirow{2}{*}{ Life Form } & \multirow{2}{*}{ Parts Used } & \multirow{2}{*}{$\begin{array}{l}\text { Preparation and } \\
\text { Application }\end{array}$} & \multirow{2}{*}{ Diseases Cured } & \multirow{2}{*}{$\begin{array}{l}\text { Other Economic } \\
\text { Applications }\end{array}$} & \multicolumn{3}{|c|}{ Ethnic Groups } \\
\hline & & & & & & & Balti & Brokpa & Beda \\
\hline $\begin{array}{l}\text { Tanacetum gracile } \\
\text { Hook.f. \& Thomson. } \\
\text { (Tan-gra) } \\
\text { Asteraceae } \\
\text { SMH-146 }\end{array}$ & $\begin{array}{l}\text { Khamchu/ } \\
\text { Daisy }\end{array}$ & Herb & $\begin{array}{l}\text { Leaves } \\
\text { Flowers }\end{array}$ & $\begin{array}{l}\text { Leaves and flowers are } \\
\text { shade-dried. } \\
\text { Flowers are shade-dried } \\
\text { and covered with a fine } \\
\text { cotton cloth. }\end{array}$ & $\longrightarrow$ & $\begin{array}{l}\text { Leaves are a good source } \\
\text { of oil. } \\
\text { Flowers are fragrant and } \\
\text { a good source of oil. }\end{array}$ & $\mathrm{N}$ & Y & $\mathrm{Y}$ \\
\hline $\begin{array}{l}\text { Thlaspi arvense L. } \\
\text { (Thl-arv) } \\
\text { Brassicaceae } \\
\text { SMH-170 }\end{array}$ & $\begin{array}{l}\text { Bre-ga/ } \\
\text { Field pennycress }\end{array}$ & Herb & Leaves & $\begin{array}{l}\text { Leaves are dried and } \\
\text { powdered; decoction is } \\
\text { made and taken orally. }\end{array}$ & $\begin{array}{l}\text { Pulmonary diseases, } \\
\text { kidney diseases, and } \\
\text { white discharges. }\end{array}$ & $\longrightarrow$ & $\mathrm{Y}$ & $\mathrm{Y}$ & $\mathrm{Y}$ \\
\hline $\begin{array}{c}\text { Trigonella emodi Benth. } \\
\text { (Tri-emo) } \\
\text { Fabaceae } \\
\text { SMH-147 }\end{array}$ & $\begin{array}{l}\text { Hbu-su-hang/ } \\
\text { Himalayan } \\
\text { fenugreek }\end{array}$ & Herb & $\begin{array}{l}\text { Seeds } \\
\text { Leaves }\end{array}$ & $\begin{array}{l}\text { Seeds are dried, ground, } \\
\text { mixed with turmeric to } \\
\text { form a paste, and applied } \\
\text { topically. } \\
\text { Leaves are dried and } \\
\text { powdered; decoction is } \\
\text { made and taken orally. }\end{array}$ & $\begin{array}{l}\text { Seeds are used against } \\
\text { sores and bone fractures. } \\
\text { Leaves are used to treat } \\
\text { kidney disorders. }\end{array}$ & & Y & Y & $\mathrm{N}$ \\
\hline $\begin{array}{c}\text { Urtica hyperborea Jacq. } \\
\text { ex Wedd. } \\
\text { (Urt-hyp) } \\
\text { Urticaceae } \\
\text { SMH-171 }\end{array}$ & $\begin{array}{c}\text { Rza-sot/ } \\
\text { Northern nettle }\end{array}$ & Herb & Leaves & $\begin{array}{c}\text { Leaves are dried, } \\
\text { powdered, and taken orally } \\
\text { with lukewarm water. }\end{array}$ & $\begin{array}{l}\text { Promotes digestive and } \\
\text { physical heat, and wind } \\
\text { diseases associated with } \\
\text { chronic fever. }\end{array}$ & $\begin{array}{l}\text { Leaves are used as food } \\
\text { (soup). }\end{array}$ & $\mathrm{N}$ & Y & $\mathrm{Y}$ \\
\hline $\begin{array}{l}\text { Waldheimia tomentosa } \\
\text { (Decne.) Regel. } \\
\text { (Wal-tom) } \\
\text { Asteraceae } \\
\text { SMH-114 }\end{array}$ & $\begin{array}{l}\text { Palu/ } \\
\text { Yellow berried } \\
\text { nightshade }\end{array}$ & Herb & Whole plant & $\begin{array}{l}\text { Decoction is made and } \\
\text { used orally. Fresh and } \\
\text { dried plants are used. }\end{array}$ & $\begin{array}{l}\text { Acidity, headache, } \\
\text { and arthritis. }\end{array}$ & $\begin{array}{l}\text { Whole plant is considered } \\
\text { holy in the Buddhist faith } \\
\text { and used for making } \\
\text { incense (dhoop), which is } \\
\text { used in houses and } \\
\text { religious places. } \\
\text { Plant is also used in the } \\
\text { bathing ceremony of } \\
\text { deceased persons and } \\
\text { newborn babies. }\end{array}$ & Y & Y & Y \\
\hline
\end{tabular}


The distribution of the collected plant species in the 39 families was uneven. About half of the collected plant species belonged to just six families, i.e., Asteraceae, Ranunculaceae, Fabaceae, Apiaceae, Lamiaceae, and Polygonaceae, while the remaining half belonged to 32 families. Most of the genera (19) were monotypic (Table 2). Because of their wide range of ecological amplitudes, Asteraceae adapt easily in arid and dry habitats [39,40]. Several studies have found Asteraceae to be a dominant family in surrounding areas [28,41], although Kayani et al. [36] reported Ranunculaceae as the most dominant family from the high-altitude areas of Pakistan. Similar findings were reported by Bhattarai et al. [26] from trans-Himalayan Nepal, Ijaz et al. [42] from the Pakistani Himalayas, and Kala [43] from trans-Himalayan India. Kayani et al. [36] and Abbas et al. [44] found Fabaceae and Ranunculaceae to be prominent families from the Pakistani Himalayas. Similarly, Debbarma et al. [45] reported Fabaceae as the dominant family in northeast India. However, Pala et al. [46] reported Lamiaceae as the leading family from the Eastern Himalayas, which is in line with our results. Similar species distribution patterns were observed by other ethnobotanical studies from the Himalayas $[47,48]$. The large number of therapeutic plants from the families Asteraceae, Apiaceae, Fabaceae, Lamiaceae, Ranunculaceae, and Polygonaceae is possibly due to the abundance and wider distribution of these families in this area [43]. Furthermore, according to various researchers [49-51], the members of these families have a high content of useful bioactive compounds.

\subsection{Preference Analysis}

We emphasized the numerous ethnobotanical uses of the reported species among the local communities. The results obtained through preference analysis indicated a considerable variance $\left(\chi^{2}=408.56, \mathrm{df}=7, p<0.001\right)$ in plant usage between the different communities. Medicinal use was overall the most prevalent, with $70 \%$ of use reports, followed by fodder, fuel wood, food, fragrance, dye, flavor, and oil (Figure 3a). This demonstrates that local communities prefer the traditional "Sowa-Rigpa" (ancient Indian medicinal system, which evolved in the entire trans-Himalayan region) healthcare system [52]. This also reflects the demand of the pharmaceutical industry, given the high market value for medicinal species [53,54]. Ijaz et al. [42] reported similar results from Pakistan. Haq et al. [48] also reported maximum usage of plants for medicinal purposes from the Northwestern Himalayas, followed by other ethnobotanical uses. Other studies [54-57] found similar results.

The indigenous community used different plant parts for various ethnobotanical uses (Figure $3 b)$ with a significant difference $\left(\chi^{2}=100.12, \mathrm{df}=9, p<0.001\right)$ between their usage. The results obtained through preference analysis indicated a noteworthy variance, with leaves $(27 \%)$ the most used, followed by roots, flowers, stem, fruits, whole plant, bulbs, bark, seeds, and young twigs (Figure $3 b$ ). The PCA analysis also supported our results and showed ten individual groups centered on the variations in the preference levels of plant parts usage (Figure 4). PC1 and PC2 explained 50.7\% of the parts used in the biplot, in which ten clusters of plant part usage based on species presence/absence can be identified: leaves, roots, bulbs, flowers, seeds, bark, whole plant, fruits, stem, and young twigs (Figure 4). Due to the dependence of local people on wild plant resources for daily cuisine, different plant parts are preferred according to their uses. Leaves are the main photosynthetic organs and thus contain lots of metabolites $[28,58]$. Furthermore, using leaves and aerial parts is regarded as safe as well as sustainable [59]. Roots are also known to contain a good concentration of bioactive compounds [60,61], and local shepherds, Amchis and herbal drug dealers, and other ethno-groups often prefer to use/trade the roots of plants for medicinal purposes [62]. 
a)

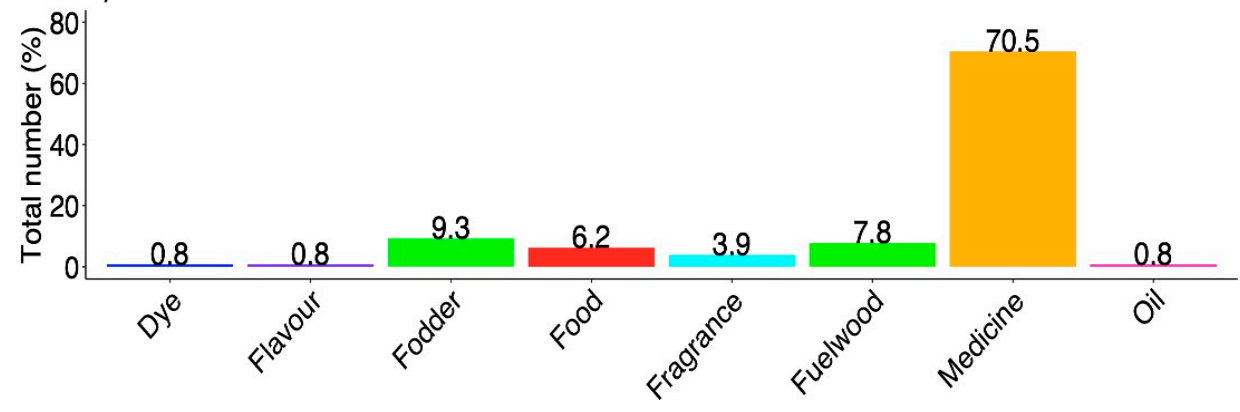

b)

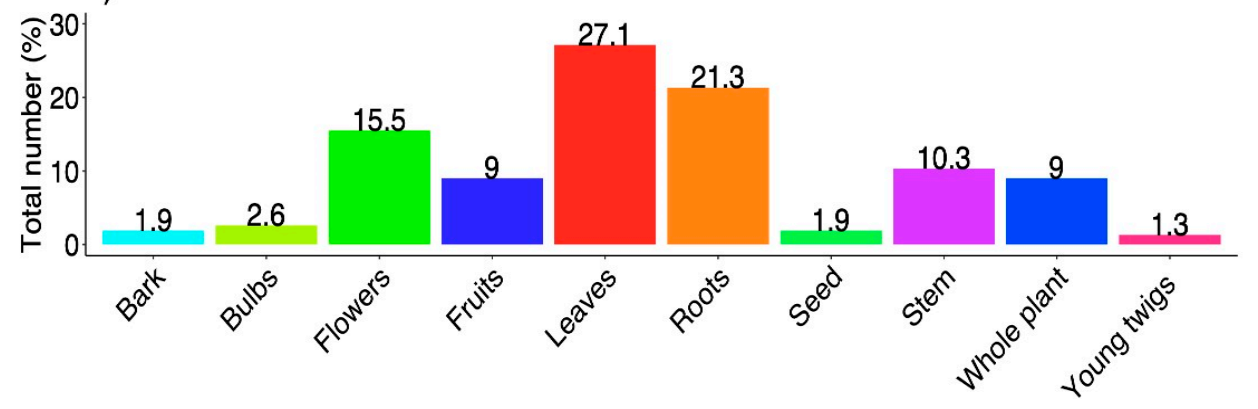

Figure 3. (a) Percentage of different ethnobotanical usages; (b) percentage of different plant parts used in the Ladakh trans-Himalayan region, India.

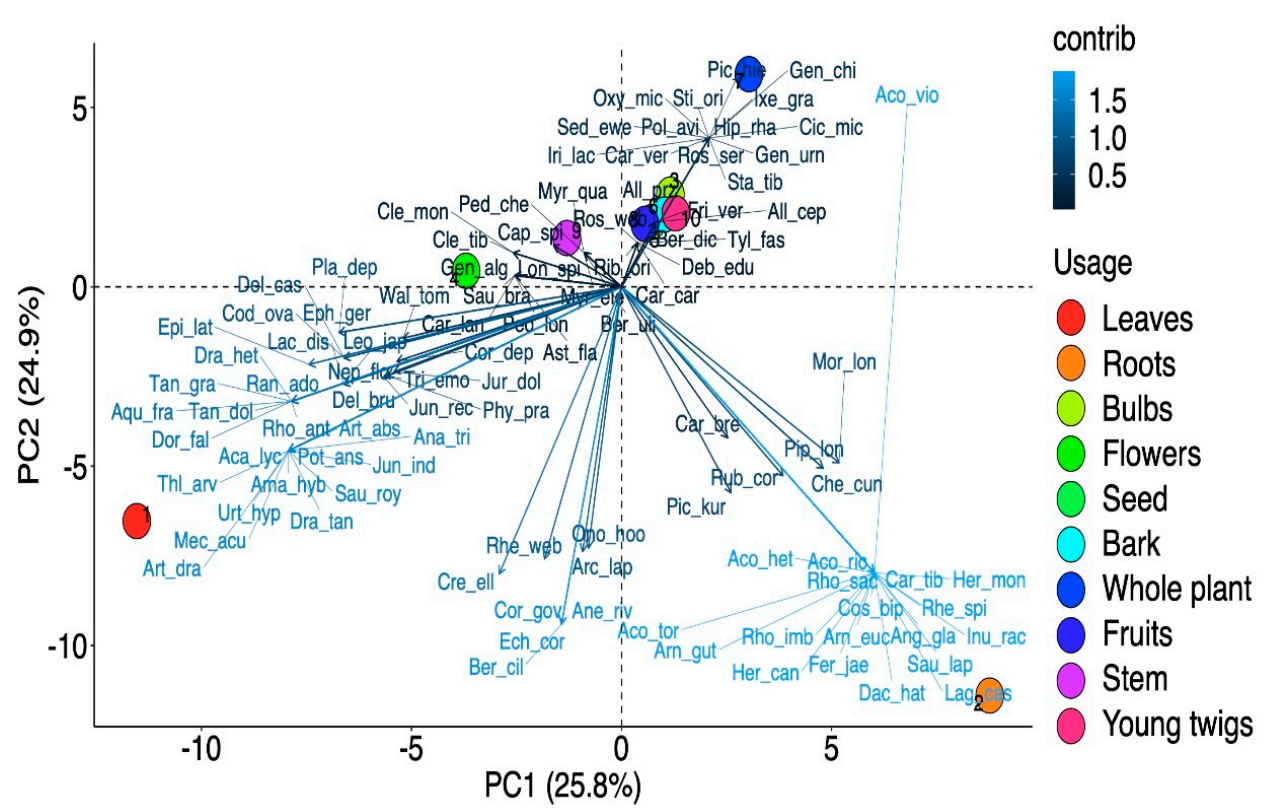

Figure 4. Principal component analysis (PCA) biplot of different part(s) usage in the Ladakh transHimalayan region, India.

The overharvesting of underground parts or whole plants should be discouraged, especially in the case of threatened species, as this practice causes elimination and dwindling of the plant's status in the wild [43,63]. Our findings are also supported by Ahmad et al. [64], Sharif et al. [65], Siddique et al. [66], Anwer et al. [67], and Manduzai et al. [68] from the Pakistan Himalayas; and Debbarma et al. [45] and Krupa et al. [69] from India. Asif et al. [28] and Haq et al. [48] from the Kashmiri Himalayas, India; Pala et al. [46] from the Eastern Himalayas; Singh et al. [70] from the Western Himalayas, India; and Tiwari et al. [71] from the Kumaun Himalayas, India. The collection of plant parts is designed depending upon the availability of plant parts in various pheno-phases following the 
Tibetan traditional calendar [72]. For example, leaves were collected in spring (April and May), flowers and mature leaves in summer (June and August), and, finally, fruits, roots, and seeds in autumn (September and November). The rural inhabitants, herders, Amchis, and elderly people were aware of plant collection timings and selective harvesting of plants for ethnobotanical usage. A similar pattern of plant part collection was reported by Lone et al. [38], Kala [72], Ghimire et al. [73], and Kala [74], from the Himalayas [38,72-74].

Wild leafy vegetables such as Allium przewalskianum, Amaranthus spinosus, Plantago depressa, and Urtica hyperborea growing close to and in human settlements were especially frequently used. The leaves of Urtica hyperborea were commonly used for making soup by the Buddhist inhabitants of Leh. It was also noted that for herbal preparations, plant parts were mostly used in dried form and the reason for this was that the dried plant parts were kept for later seasons, particularly for the winter season [48]. Most of the formulations were prepared and administered at home, like in the results of Lone et al. [38]. The local people in their respective localities were sometimes assisted by other knowledgeable people, when necessary, with no or very low charges. However, it was stated by most of the informants that they kept their knowledge of medicinal plants secret. Furthermore, they revealed that the sharing of traditional knowledge of medicinal plants may take place only with family members, mostly from parents to sons, which is one reason why, in the present study, it was documented that men have more knowledge about medicinal plants than women.

\subsection{Cross-Cultural and Religious Analysis}

The Venn diagram (Figure 5) shows that the maximum number of plant uses was reported by the Brokpa, while the Beda reported a minimum number of plant uses. The Balti and Brokpa ethnic groups showed greater similarity, whereas the least overlap was observed between Beda and Brokpa. A cross-cultural comparison of plant resources showed that 37 plants were commonly used by all ethnic groups. Gairola et al. [75] also reported on the cross-cultural usage of plants from the Himalayas. Plants common among all cultures mainly had medicinal value, although certain plants were common because of their religious uses in the two main religions (Islam and Buddhism). Some species were found in all cultures (Balti, Brokpa, and Beda).

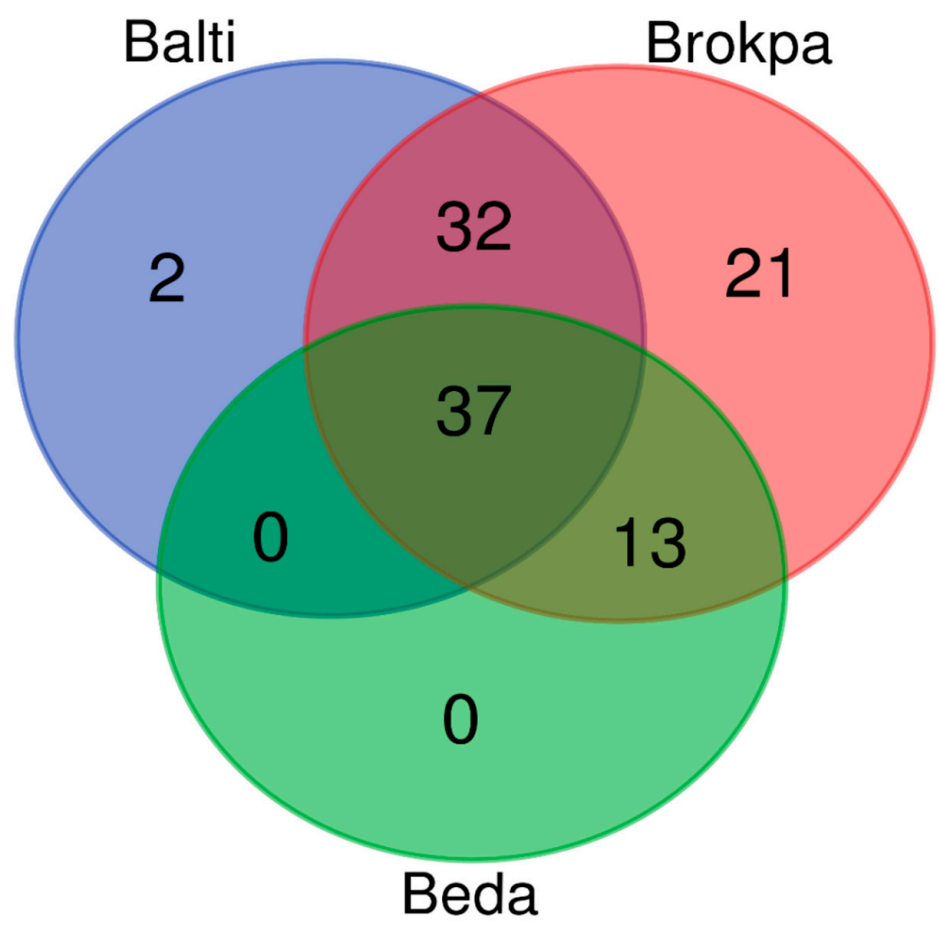

Figure 5. Venn diagram showing the overlap of ethnobotanical usage of plants in different ethnic groups in the Ladakh trans-Himalayan region, India. 
Many of the plants used in the wider region play a significant role in some cultural and religious ceremonies [76,77]. Delphinium brunonianum (Ba-ru-ra/Ladar) was used by local healers in dealing with evil spirits. Waldheimia tomentosa (Palu) is an aromatic holy plant which was used as incense (locally known as dhoop) in houses and religious places on auspicious religious and cultural days. Palu was also used in the bathing ceremony for deceased persons and in the baby shower of newborns in the Buddhist faith. The dried leaves of Waldheimia tomentosa were added to hot water and then used during bathing. Similarly, it was found that Juniperus indica (Shukpa) was used as incense by Buddhists in their monasteries and in religious and marriage events, as well as in dealing with nightmares. The dried leaves of this plant were burned in a mud pot to produce smoke, which was kept in front of people who were dealing with bad dreams, especially by Muslims. Plant species such as Codonopsis ovata and Cremanthodium ellisii were used to fend off evil spirits. The seeds of Datura stramonium (Esman) were used by the Balti tribe for dealing with evil spirits. Branches of Salix pycnostachya Andersson (Malchang) were used as pillows for corpses in graves (Muslim faith). A similar usage of plant resources for religious and ritual beliefs was reported by Amjad et al. [78] from Pakistan and Sharma et al. [79] from Assam, India.

\subsection{Classification of Ethnobotanical Usage}

Cluster analysis elucidated three clusters of different ethnobotanical uses based on floristic similarity. The first cluster included food and medicinal plants, the second included dye and flavor plants, and the third included plants used for fragrance, oil, fuel wood, and fodder (Figure 6). Species such as Amaranthus spinosus, Allium humile, and Allium przewalskianum, having both medicinal and food value, were grouped in the first cluster. Plants such as Artemisia absinthium and Oxytropis microphylla, used as dyes as well as flavoring agents, fell into the second cluster. Plants such as Caragana versicolor and Hippophae rhamnoides, used as fodder as well as fuel wood and oil sources, formed a separate, third cluster. The principal component analysis (PCA) also supported these results, showing distinct use clusters based on variations in the preference levels (Figure 7). The PCA correlated the most important components with other underlying variables. PC1 and PC2 explained $89.2 \%$ of the provisioning services in the biplot, in which five clusters of ethnobotanical usage based on species presence/absence can be identified: food, medicine, fuel wood, fodder, and fragrance, oil, dye, and flavor. Similar classifications were found in previous studies. For example, Asif et al. [28] reported five groups of wild plants from tribal communities in the tehsil of Karnah (Jammu and Kashmir), India. Haq et al. [48] classified the wild plants of district Reasi into four plant usage groups. Rivera et al. [80] observed eight major clusters in the mountains of Castilla-La Mancha (Spain). Similarly, multivariate analysis was used by Balemie and Kebebew [81], Leduc et al. [82], Caneva et al. [83], and Haq et al. [84] for quantitative ethnobiological approaches in their studies.

\subsection{Important Medicinal Plant Species, Their Local Uses, and Trade Status}

The local inhabitants collected most plants for self-use (65\%) or for income earning $(35 \%)$. Every single medicinal plant found in the study area is valuable in the local healthcare system, although some have especially high significance value in the traditional "Sowa-Rigpa" healthcare system, e.g., Aconitum heterophyllum, Aconitum violaceum, Arnebia guttata, Arnebia euchroma, Aster flaccidus, Bergenia stracheyi, Corydalis govaniana, Dactylorhiza hatagirea, Gentiana algida, Hippophae rhamnoides, Inula racemosa, Jurinea dolomiaea, Meconopsis aculeata, Picrorhiza kurroa, Rhododendron anthopogon, Rheum webbianum, Rheum spiciforme, Saussurea bracteata, Saussurea lappa, and Vincetaxicum caneces. Allium przewalskianum, Waldheimia tomentosa, and Juniperus indica (Shukpa) were found to be commonly used by the Ladakhi people as a source of income at the local level (Leh market). Similarly, it was found that plants such as Allium przewalskianum, Amaranthus spinosus, Allium humile, Plantago depressa, and Urtica hyperborea were mostly used as food, and Hippophae rhamnoides was used to make juice in the study area. Plants, in addition to playing a role in treating various 
ailments [85-87], also deliver tangible economic benefits to indigenous communities, as in other regions [88]. The sustainable utilization and management of wild resources can act as a strategy to boost livelihood generation and food security and aid in poverty alleviation. Khan et al. [89], while carrying out a study on the indigenous communities of Ladakh, reported that 14.05 percent of the households surveyed were involved in medicinal plant collection, while only 8.11 percent were involved in marketing. The medicinal plants generated a total income of $274,034.40$ annum $^{-1}$ at 1481.27 household $^{-1}$ annum $^{-1}$ and 514.09 man-days annum $^{-1}$ in the sampled population, with an average employment opportunity of 0.35 man-days household ${ }^{-1}$ annum $^{-1}$. In terms of subsistence and income generation, medicinal plants play an important role in aboriginal people's livelihood support.

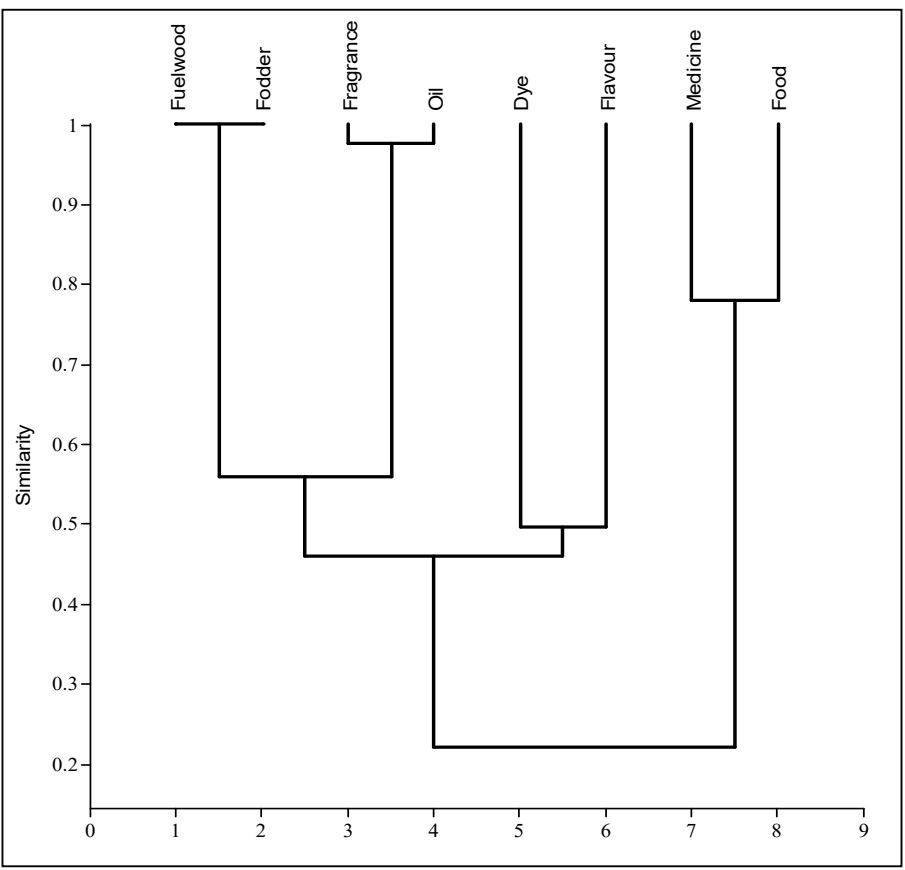

Figure 6. Cluster diagram of the different provisioning services based on plant usage patterns in the Ladakh trans-Himalayan region, India.

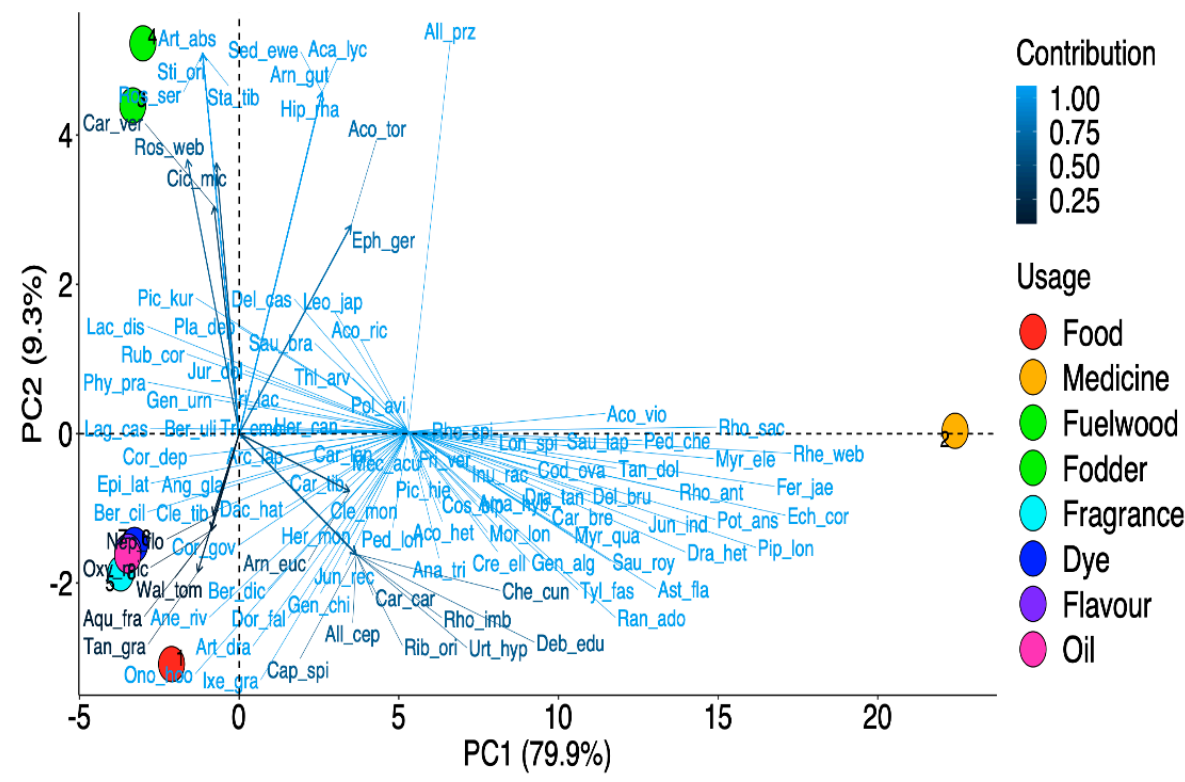

Figure 7. Principal component analysis (PCA) biplot of different provisioning services in the Ladakh trans-Himalayan region, India. 


\section{Conclusions}

The ethnobotanical results of this study clearly demonstrate that the traditional knowledge of medicinal plants is mainly the asset of elders. A total of 105 plant species belonging to 82 genera and 39 families were documented. The results indicated that the most prominent family is Asteraceae. The plant part most used are leaves. Medicinal uses are the most prevalent, with $70 \%$ of use reports, followed by fodder and fuel wood. Delphinium brunonianum, Waldheimia tomentosa, and Juniperus indica play a significant role in the cultural and religious ritual aspects. The local people collect most plants (65\%) for self-consumption, while the rest $(35 \%)$ are sold in markets as a source of income. The comparative analysis with previously published works showed similarities with our data. The results clearly indicate a real risk of progressive loss of traditional knowledge. In this study, some plants were reported for the first time for their ethnomedicinal use. These should be assessed for phytochemical composition and pharmacological activities. Further research on conservation strategies needs to be conducted.

Author Contributions: Conceptualization, S.M.H. and U.Y.; methodology, S.M.H.; software, E.S.C.; validation R.W.B., A.M.A. and S.M.H.; formal analysis, S.M.H. and E.S.C.; investigation, S.M.H. and M.H.; resources, S.M.H.; data curation, S.M.H. and M.H.; writing, S.M.H., U.Y. and M.H.; original draft preparation, R.W.B., A.M.A. and I.U.R.; review and editing, R.W.B., A.M.A. and I.U.R.; visualization, S.M.H.; supervision, R.W.B., A.M.A. and I.U.R.; review and editing, A.H., E.F.A., M.A.A., A.A.A., M.A., S.U.R. and F.I. All authors have read and agreed to the published version of the manuscript.

Funding: The authors would like to extend their sincere appreciation to the Researchers Supporting Project (No. RSP-2021/134), King Saud University, Riyadh, Saudi Arabia.

Institutional Review Board Statement: Not applicable.

Informed Consent Statement: Informed consent was obtained from all subjects involved in the study.

Acknowledgments: Thanks are due to the local people of Ladakh for sharing the ethnobotanical information and cooperating during the surveys and interviews. The authors are grateful to all those who directly or indirectly helped them during the study. The authors would like to extend their sincere appreciation to the Researchers Supporting Project (No. RSP-2021/134), King Saud University, Riyadh, Saudi Arabia.

Conflicts of Interest: The authors declare no conflict of interest.

\section{References}

1. Seppelt, R.; Dormann, C.F.; Eppink, F.V.; Lautenbach, S.; Schmidt, S. A Quantitative Review of Ecosystem Service Studies: Approaches, Shortcomings and the Road Ahead. J. Appl. Ecol. 2011, 48, 630-636. [CrossRef]

2. Jordan, S.J.; Hayes, S.E.; Yoskowitz, D.; Smith, L.M.; Summers, J.K.; Russell, M.; Benson, W.H. Accounting for Natural Resources and Environmental Sustainability: Linking Ecosystem Services to Human Well-Being; ACS Publications: Washington, DC, USA, 2010.

3. Butler, C.D.; Oluoch-Kosura, W. Linking Future Ecosystem Services and Future Human Well-Being. Ecol. Soc. 2006, 11, 30. [CrossRef]

4. O'Neill, B.C.; Kriegler, E.; Ebi, K.L.; Kemp-Benedict, E.; Riahi, K.; Rothman, D.S.; van Ruijven, B.J.; van Vuuren, D.P.; Birkmann, J.; Kok, K. The Roads Ahead: Narratives for Shared Socioeconomic Pathways Describing World Futures in the 21st Century. Glob. Environ. Chang. 2017, 42, 169-180. [CrossRef]

5. Pereira, E.; Queiroz, C.; Pereira, H.M.; Vicente, L. Ecosystem Services and Human Well-Being: A Participatory Study in a Mountain Community in Portugal. Ecol. Soc. 2005, 10, 14. [CrossRef]

6. Perrino, E.V.; Valerio, F.; Gannouchi, A.; Trani, A.; Mezzapesa, G. Ecological and Plant Community Implication on Essential Oils Composition in Useful Wild Officinal Species: A Pilot Case Study in Apulia (Italy). Plants 2021, 10, 574. [CrossRef]

7. Roy, V. Integrating Indigenous Systems of Medicines in the Healthcare System in India: Need and Way Forward. In Herbal Medicine in India; Springer: Berlin/Heidelberg, Germany, 2020; pp. 69-87.

8. Allkin, B. Useful medicines. In State of the World's Plants 2017; Willis, K.J., Ed.; The Board of Trustees of the Royal Botanic Gardens, Royal Botanic Gardens, Kew: London, UK, 2017; pp. 22-29.

9. Molnár, Z.; Berkes, F. Role of Traditional Ecological Knowledge in Linking Cultural and Natural Capital in Cultural Landscapes. In Reconnecting Natural and Cultural Capital: Contributions from Science and Policy; Paracchini, M.L., Zingari, P.C., Blasi, C., Eds.; Publications Office of the European Union: Luxembourg, 2018; pp. 183-193. 
10. Jadhav, V.D.; Mahadkar, S.D.; Valvi, S.R. Documentation and Ethnobotanical Survey of Wild Edible Plants from Kolhapur District. Rec. Res. Sci. Technol. 2012, 3, 58-63.

11. Jianchu, X.; Mikesell, S. Indigenous Knowledge for Sustainable Livelihoods and Resources Governance in MMSEA Region. In Proceedings of the III Symposium on MMSEA, Lijiang, China, 25-28 August 2002; pp. 1-7.

12. Dhondrup, W.; Tidwell, T.; Wang, X.; Tso, D.; Dhondrup, G.; Luo, Q.; Wangmo, C.; Kyi, T.; Liu, Y.; Meng, X. Tibetan Medical Informatics: An Emerging Field in Sowa Rigpa Pharmacological \& Clinical Research. J. Ethnopharmacol. 2020, $250,112481$. [PubMed]

13. Ballabh, B.; Chaurasia, O.P. Traditional Medicinal Plants of Cold Desert Ladakh-Used in Treatment of Cold, Cough and Fever. J. Ethnopharmacol. 2007, 112, 341-349. [CrossRef]

14. Ballabh, B.; Chaurasia, O.P. Medicinal Plants of Cold Desert Ladakh Used in the Treatment of Stomach Disorders. Ind. J. Trad. Knowl. 2009, 8, 185-190.

15. Haq, S.M.; Calixto, E.S.; Yaqoob, U.; Ahmed, R.; Mahmoud, A.H.; Bussmann, R.W.; Mohammed, O.B.; Ahmad, K.; Abbasi, A.M. Traditional Usage of Wild Fauna among the Local Inhabitants of Ladakh, Trans-Himalayan Region. Animals 2020, 10, 2317. [CrossRef]

16. Kala, C.P.; Mathur, V.B. Patterns of Plant Species Distribution in the Trans-Himalayan Region of Ladakh, India. J. Veg. Sci. 2002, 13, 751-754. [CrossRef]

17. Ethnobiology, I.S. International Society of Ethnobiology Code of Ethics (with 2008 Additions). 2006. Available online: https: //www.ethnobiology.net/what-we-do/core-programs/ise-ethics-program/code-of-ethics/ (accessed on 28 October 2020).

18. Sharma, U.K.; Pegu, S. Ethnobotany of Religious and Supernatural Beliefs of the Mising Tribes of Assam with Special Reference to the'Dobur Uie'. J. Ethnobiol. Ethnomed. 2011, 7, 1-13. [CrossRef] [PubMed]

19. Singh, A.; Lal, M.; Samant, S.S. Diversity, Indigenous Uses and Conservation Prioritization of Medicinal Plants in Lahaul Valley, Proposed Cold Desert Biosphere Reserve, India. Int. J. Biodiv. Sci. Manag. 2009, 5, 132-154. [CrossRef]

20. Chaurasia, O.P.; Khatoon, N.; Singh, S.B. Field Guide Plant. Biodiversity of Ladakh; WWF-India: New Delhi, India, 2008.

21. Sorensen, T.A. Method of establishing groups of equal amplitude in plant sociology based on similarity of species content and its application to analyses of the vegetation on Danish commons. Biol. Skr. 1948, 5, 1-34.

22. Dalirsefat, S.B.; da Silva Meyer, A.; Mirhoseini, S.Z. Comparison of Similarity Coefficients Used for Cluster Analysis with Amplified Fragment Length Polymorphism Markers in the Silkworm, Bombyx Mori. J. Insect Sci. 2009, 9, 1-8. [CrossRef]

23. Jari Oksanen, F.; Blanchet, G.; Friendly, M.; Kindt, R.; Legendre, P.; McGlinn, D.; Wagner, H. Vegan: Community Ecology Package. R Package Version 2.5-3. 2018. Available online: https://cran.r-project.org/web/packages/vegan/index.html (accessed on 22 January 2021).

24. R Core Team. A Language and Environment for Statistical Computing; R Foundation for Statistical Computing: Vienna, Austria, 2020.

25. Gu, Z.; Gu, L.; Eils, R.; Schlesner, M.; Brors, B. Circlize Implements and Enhances Circular Visualization in R. Bioinformat 2014, 30, 2811-2812. [CrossRef]

26. Bhattarai, S.; Chaudhary, R.P.; Quave, C.L.; Taylor, R.S. The Use of Medicinal Plants in the Trans-Himalayan Arid Zone of Mustang District, Nepal. J. Ethnobiol. Ethnomed. 2010, 6, 1-11. [CrossRef]

27. Rashid, S.; Ahmad, M.; Zafar, M.; Sultana, S.; Ayub, M.; Khan, M.A.; Yaseen, G. Ethnobotanical Survey of Medicinally Important Shrubs and Trees of Himalayan Region of Azad Jammu and Kashmir, Pakistan. J. Ethnopharmacol. 2015, 166, 340-351. [CrossRef] [PubMed]

28. Asif, M.; Haq, S.M.; Yaqoob, U.; Hassan, M.; Jan, H.A. A Preliminary Study on the Ethno-Traditional Medicinal Plant Usage in Tehsil "Karnah" of District Kupwara (Jammu and Kashmir) India. Ethnobot. Res. Appl. 2021, 21, 1-14.

29. Bhatia, H.; Sharma, Y.P.; Manhas, R.K.; Kumar, K. Ethnomedicinal Plants Used by the Villagers of District Udhampur, J\&K, India. J. Ethnopharmacol. 2014, 151, 1005-1018. [PubMed]

30. Emmanuel, M.M.; Didier, D.S. Medicinal Plant Knowledge of Ethnic Groups in Douala Town, Cameroon. Am. J. Food Nutr. 2011, 1, 178-184. [CrossRef]

31. Ambu, G.; Chaudhary, R.P.; Mariotti, M.; Cornara, L. Traditional Uses of Medicinal Plants by Ethnic People in the Kavrepalanchok District, Central Nepal. Plants 2020, 9, 759. [CrossRef]

32. Awan, M.R.; Jamal, Z.; Khan, A. Ethno-Botanical Studies of Economically Important Plants from Mountainous Region of Gilgit-Baltistan, Pakistan. Sci. Tech. Dev. 2013, 32, 308-318.

33. Mulk, K.S.; Page, S.; Ahmad, H.; Shaheen, H.; Ullah, Z.; Ahmad, M.; Harper, D. Medicinal Flora and Ethnoecological Knowledge in the Naran Valley, Western Himalaya, Pakistan. J. Ethnobiol. Ethnomed. 2013, 9, 10.

34. Ajaib, M.; Islam, A.; Sidiqui, M.F. A Contribution to Ethnobotanical Study of Wild Plants of Tehsil Jatlan Azad Jammu \& Kashmir. FUUAST J. Biol. 2016, 6, 247-256.

35. Rana, S.K.; Rawat, G.S. Ethnobotanical Survey of Medicinal Plants for Assessment of Their Conservation Status in Chenab Valley, J\&K. In Proceedings of the 1st Himalayan Researchers Consortium, Dehradun, India, 26-27 April 2019 ; Volume 1.

36. Kayani, S.; Ahmad, M.; Sultana, S.; Shinwari, Z.K.; Zafar, M.; Yaseen, G.; Hussain, M.; Bibi, T. Ethnobotany of Medicinal Plants among the Communities of Alpine and Sub-Alpine Regions of Pakistan. J. Ethnopharmacol. 2015, 164, 186-202. [CrossRef]

37. Haider, A.; Qaiser, M. The Enthnobotany of Chitral valley, Pakistan with particular reference to medicinal Plants. Pak. J. Bot. 2009, 41, 2009-2041. 
38. Lone, P.A.; Bhardwaj, A.K. Traditional Herbal Based Disease Treatment in Some Rural Areas of Bandipora District of Jammu and Kashmir, India. As. J. Pharm. Clin. Res. 2013, 6, 162-171.

39. Mitra, S.; MuKherjee, S.K. Asteraceae of India: Its Diversity and Phytogeographical Affinity. In Plant Biodiversity: Monitoring, Assessment and Conservation; Ansari, A.A., Gill, S.S., Abbas, Z.K., Naeem, M., Eds.; CAB International: Wallingford, UK, 2016; pp. 36-70. [CrossRef]

40. Haq, S.M.; Hamid, M.; Lone, F.A.; Singh, B. Himalayan Hotspot with Alien Weeds: A Case Study of Biological Spectrum, Phenology, and Diversity of Weedy Plants of High Altitude Mountains in District Kupwara of J\&K Himalaya, India. Proc. Natl. Acad. Sci. India Sect. B Biol. Sci. 2021, 91, 139-152.

41. Hamid, A.; Raina, A.K. Ethnobotanical Uses of Plants in and around Kanji Wildlife Sanctuary, North West Himalaya. Int. J. Sci. Res. 2014, 3, 538-545.

42. Ijaz, F.; Rahman, I.U.; Iqbal, Z.; Alam, J.; Ali, N.; Khan, S.M. Ethno-ecology of the healing Forests of Sarban Hills, Abbottabad, Pakistan: An economic and medicinal appraisal. In Plant and Human Health; Springer: Berlin/Heidelberg, Germany, 2018; Volume 1, pp. 675-706.

43. Kala, C.P. Medicinal Plants of the High Altitude Cold Desert in India: Diversity, Distribution and Traditional Uses. J. Biodiv. Sci. Ecosyst. Serv. Manag. 2006, 2, 43-56. [CrossRef]

44. Abbas, Z.; Khan, S.M.; Alam, J.; Khan, S.W.; Abbasi, A.M. Medicinal Plants Used by Inhabitants of the Shigar Valley, Baltistan Region of Karakorum Range-Pakistan. J. Ethnobiol. Ethnomed. 2017, 13, 1-15. [CrossRef] [PubMed]

45. Debbarma, M.; Pala, N.A.; Kumar, M.; Bussmann, R.W. Traditional Knowledge of Medicinal Plants in Tribes of Tripura in Northeast, India. Afr. J. Tradit. Comp. Altern. Med. 2017, 14, 156-168. [CrossRef]

46. Pala, N.A.; Sarkar, B.C.; Shukla, G.; Chettri, N.; Deb, S.; Bhat, J.A.; Chakravarty, S. Floristic Composition and Utilization of Ethnomedicinal Plant Species in Home Gardens of the Eastern Himalaya. J. Ethnobiol. Ethnomed. 2019, 15, 1-16. [CrossRef] [PubMed]

47. Sharma, J.; Raina, A.K. Medicinal Plants of Lamberi Forest Range, Rajouri, J\&K, India. India Environ. Conserv. 2016, 17, 153-160.

48. Haq, S.M.; Singh, B. Ethnobotany as a Science of Preserving Traditional Knowledge: Traditional Uses of Wild Medicinal Plants from District Reasi, J\&K (Northwestern Himalaya), India. In Botanical Leads for Drug Discovery; Springer: Berlin/Heidelberg, Germany, 2020; pp. 277-293.

49. Valerio, F.; Mezzapesa, G.N.; Ghannouchi, A.; Mondelli, D.; Logrieco, A.F.; Perrino, E.V. Characterization and Antimicrobial Properties of Essential Oils from Four Wild Taxa of Lamiaceae Family Growing in Apulia. Agronomy 2021, 11, 1431. [CrossRef]

50. Marchioni, I.; Najar, B.; Ruffoni, B.; Copetta, A.; Pistelli, L.; Pistelli, L. Bioactive Compounds and Aroma Profile of Some Lamiaceae Edible Flowers. Plants 2020, 9, 691. [CrossRef]

51. Derouich, M.; Bouhlali, E.D.T.; Bammou, M.; Hmidani, A.; Sellam, K.; Alem, C. Bioactive Compounds and Antioxidant, Antiperoxidative, and Antihemolytic Properties Investigation of Three Apiaceae Species Grown in the Southeast of Morocco. Scientifica 2020, 2020, 1-10. [CrossRef]

52. Blaikie, C. Currents of Tradition in Sowa Rigpa Pharmacy. East Asian Sci. Technol. Soc. Int. J. 2013, 7, 425-451. [CrossRef]

53. Shinwari, Z.K.; Gilani, S.S. Sustainable Harvest of Medicinal Plants at Bulashbar Nullah, Astore (Northern Pakistan). J. Ethnopharmacol. 2003, 84, 289-298. [CrossRef]

54. Naveed, A.; Abdur, R.; Waheed, M.; Bergmeier, E. Diversity and Use of Ethno-Medicinal Plants in the Region of Swat, North Pakistan. J. Ethnobiol. Ethnomed. 2013, 9, 25.

55. Sharma, P.; Patti, P.; Agnihotry, A. Ethnobotanical and Ethnomedicinal Uses of Floristic Diversity in Murari Devi and Surrounding Areas of Mandi District in Himachal Pradesh, India. Pak. J. Bio. Sci. 2013, 16, 451-468. [CrossRef] [PubMed]

56. Begum, S.; AbdEIslam, N.M.; Adnan, M.; Tariq, A.; Yasmin, A.; Hameed, R. Ethnomedicines of Highly Utilized Plants in the Temperate Himalayan Region. Afr. J. Tradit. Comp. Alernt. Med. 2014, 11, 132-142. [CrossRef]

57. Ijaz, F.; Iqbal, Z.; Rahman, I.U.; Alam, J.; Khan, S.M.; Shah, G.M.; Khan, K.; Afzal, A. Investigation of traditional medicinal floral knowledge of Sarban Hills, Abbottabad, KP, Pakistan. J. Ethnopharmacol. 2016, 179, 208-233. [CrossRef] [PubMed]

58. Vázquez-Hernández, M.C.; Parola-Contreras, I.; Montoya-Gómez, L.M.; Torres-Pacheco, I.; Schwarz, D.; Guevara-González, R.G. Eustressors: Chemical and Physical Stress Factors Used to Enhance Vegetables Production. Sci. Hortic. 2019, 250, $223-229$. [CrossRef]

59. Jan, H.A.; Jan, S.; Bussmann, R.W.; Ahmad, L.; Wali, S.; Ahmad, N. Ethnomedicinal Survey of the Plants Used for Gynecological Disorders by the Indigenous Community of District Buner, Pakistan. Ethnobot. Res. Appl. 2020, 19, 1-18. [CrossRef]

60. Adhikari, M.; Thapa, R.; Kunwar, R.M.; Devkota, H.P.; Poudel, P. Ethnomedicinal Uses of Plant Resources in the Machhapuchchhre Rural Municipality of Kaski District, Nepal. Medicines 2019, 6, 69. [CrossRef]

61. Yousuf, S.; Haq, S.M.; Rasool, A.; Zulfajri, M.; Hanafiah, M.M.; Nafees, H.; Tasneem, S.; Mahboob, M. Evaluation of Antidepressant Activity of Methanolic and Hydroalcoholic Extracts of Acorus Calamus L. Rhizome through Tail Suspension Test and Forced Swimming Test of Mice. J. Trad. Chin. Med. Sci. 2020, 7, 301-307. [CrossRef]

62. Pandey, A.; Chandra Sekar, K.; Joshi, B.; Rawal, R.S. Threat Assessment of High-Value Medicinal Plants of Cold Desert Areas in Johar Valley, Kailash Sacred Landscape, India. Plant Biosyst. 2019, 153, 39-47. [CrossRef]

63. Rathore, S.; Debnath, P.; Kumar, R. Kuth Saussurea costus (Falc.) Lipsch.: A Critically Endangered Medicinal Plant from Himalaya. J. Appl. Res. Med. Arom. 2020, 20, 100277. [CrossRef] 
64. Ahmad, K.S.; Hamid, A.; Nawaz, F.; Hameed, M.; Ahmad, F.; Deng, J.; Akhtar, N.; Wazarat, A.; Mahroof, S. Ethnopharmacological Studies of Indigenous Plants in Kel Village, Neelum Valley, Azad Kashmir, Pakistan. J. Ethnobiol. Ethnomed. 2017, 13, 1-16. [CrossRef]

65. Sharif, A.; Asif, H.; Younis, W.; Riaz, H.; Bukhari, I.A.; Assiri, A.M. Indigenous Medicinal Plants of Pakistan Used to Treat Skin Diseases: A Review. Chin. Med. 2018, 13,1-26.

66. Siddique, Z.; Shah, G.M.; Ahmed, H.M.; Nisa, S.; Khan, A.; Idrees, M.; Naz, S.; Waqas Hassan, S.; Mohiuddin, M. Ethnophytotherapy Practices for Wound Healing among Populations of District Haripur, KPK, Pakistan. Evid. Based Comp. Altern. Med. 2019, 2019, 4591675. [CrossRef] [PubMed]

67. Anwer, Z.; Shabbir, S.; Iram, T.; Tariq, S.; Murad, H. Ethnobotanical Study of Wild Flora of Haroonabad, District Bahawalnagar, Punjab, Pakistan. Eur. J. Biol. 2020, 5, 41-62. [CrossRef]

68. Manduzai, A.K.; Abbasi, A.M.; Khan, S.M.; Abdullah, A.; Prakofjewa, J.; Amini, M.H.; Amjad, M.S.; Cianfaglione, K.; Fontefrancesco, M.F.; Soukand, R. The Importance of Keeping Alive Sustainable Foraging Practices: Wild Vegetables and Herbs Gathered by Afghan Refugees Living in Mansehra District, Pakistan. Sustainability 2021, 13, 1500. [CrossRef]

69. Krupa, J.; Sureshkumar, J.; Silambarasan, R.; Priyadarshini, K.; Ayyanar, M. Integration of Traditional Herbal Medicines among the Indigenous Communities in Thiruvarur District of Tamil Nadu, India. J. Ayurved. Integr. Med. 2019, 10, 32-37. [CrossRef] [PubMed]

70. Singh, B.; Kishor, A.; Singh, B. Indigenous Plant Knowledge for Human Health Care from Jasrota Wildlife Sanctuary (Western Himalaya), India. In Botanical Leads for Drug Discovery; Springer: Berlin/Heidelberg, Germany, 2020; pp. 363-387.

71. Tiwari, D.; Sah, A.N.; Bawari, S.; Bussmann, R.W. Ethnobotanical Investigations on Plants Used in Folk Medicine by Native People of Kumaun Himalayan Region of India. Ethnobot. Res. Appl. 2020, 20, 1-35. [CrossRef]

72. Kala, C.P. Conservation of Nature and Natural Resources through Spirituality. Appl. Ecol. Environ. Sci. 2017, 5, 24-34.

73. Ghimire, S.K.; McKey, D.; Aumeeruddy-Thomas, Y. Heterogeneity in Ethnoecological Knowledge and Management of Medicinal Plants in the Himalayas of Nepal: Implications for Conservation. Ecol. Soc. 2004, 9, 6. [CrossRef]

74. Kala, C.P. Ethnobotanical and Ecological Approaches for Conservation of Medicinal and Aromatic Plants. In Proceedings of the IV International Symposium on Breeding Research on Medicinal and Aromatic Plants-ISBMAP2009 860, Ljubljana, Slovenia, 17-21 June 2009; pp. 19-26.

75. Gairola, S.; Sharma, J.; Bedi, Y.S. A Cross-Cultural Analysis of Jammu, Kashmir and Ladakh (India) Medicinal Plant Use. J. Ethnopharmacol. 2014, 155, 925-986. [CrossRef]

76. Dogan, Y. Wild Edible Plants: From the Past to the Future. Austin Food Sci. 2016, 1, 1006.

77. Chauhan, S.H.; Yadav, S.; Takahashi, T.; Luczaj, L.; D'Cruz, L.; Okada, K. Consumption Patterns of Wild Edibles by the Vasavas: A Case Study from Gujarat, India. J. Ethnobiol. Ethnomed. 2018, 14, 1-20. [CrossRef] [PubMed]

78. Amjad, M.S.; Qaeem, M.F.; Ahmad, I.; Khan, S.U.; Chaudhari, S.K.; Zahid Malik, N.; Shaheen, H.; Khan, A.M. Descriptive Study of Plant Resources in the Context of the Ethnomedicinal Relevance of Indigenous Flora: A Case Study from Toli Peer National Park, Azad Jammu and Kashmir, Pakistan. PLoS ONE 2017, 12, e0171896.

79. Sharma, U.K.; Pegu, S.; Hazarika, D.; Das, A. Medico-Religious Plants Used by the Hajong Community of Assam, India. J. Ethnopharmacol. 2012, 143, 787-800. [CrossRef]

80. Rivera, D.; Obón, C.; Inocencio, C.; Heinrich, M.; Verde, A.; Fajardo, J.; Palazón, J.A. Gathered Food Plants in the Mountains of Castilla-La Mancha (Spain): Ethnobotany and Multivariate Analysis. Econ. Bot. 2007, 61, 269-289. [CrossRef]

81. Balemie, K.; Kebebew, F. Ethnobotanical Study of Wild Edible Plants in Derashe and Kucha Districts, South Ethiopia. J. Ethnobiol. Ethnomed. 2006, 2, 1-9. [CrossRef] [PubMed]

82. Leduc, C.; Coonishish, J.; Haddad, P.; Cuerrier, A. Plants Used by the Cree Nation of Eeyou Istchee (Quebec, Canada) for the Treatment of Diabetes: A Novel Approach in Quantitative Ethnobotany. J. Ethnopharmacol. 2006, 105, 55-63. [CrossRef]

83. Caneva, G.; Traversetti, L.; Sujarwo, W.; Zuccarello, V. Sharing Ethnobotanical Knowledge in Traditional Villages: Evidence of Food and Nutraceutical "Core Groups" in Bali, Indonesia. Econ. Bot. 2017, 71, 303-313. [CrossRef]

84. Haq, S.M.; Calixto, E.S.; Singh, B. Investigation of the traditional knowledge of economically important plants in proper Neelum Valley, District Bandipora, Jammu \& Kashmir, North-Western Himalaya, India. In Plants of Novel Drug Molecules: Ethnobotany to Ethnopharmacology; Singh, B., Sharma, Y.P., Eds.; New India Publishing Agency: Delhi, India, 2020; pp. $287-302$.

85. Rahman, I.U.; Ijaz, F.; Afzal, A.; Iqbal, Z.; Ali, N.; Khan, S.M. Contributions to the phytotherapies of digestive disorders: Traditional knowledge and cultural drivers of Manoor Valley, Northern Pakistan. J. Ethnopharmacol. 2016, 192, 30-52. [CrossRef]

86. Rahman, I.U.; Afzal, A.; Iqbal, Z.; Hart, R.; Abd_Allah, E.F.; Hashem, A.; Alsayed, M.F.; Ijaz, F.; Shah, M.; Bussmann, R.W.; et al. Herbal teas and drinks: Folk medicine of the Manoor valley, Lesser Himalaya, Pakistan. Plants 2019, 8, 581. [CrossRef]

87. Rahman, I.U.; Ijaz, F.; Iqbal, Z.; Afzal, A.; Ali, N.; Afzal, M.; Khan, M.A.; Muhammad, S.; Qadir, G.; Asif, M. A novel survey of the ethno medicinal knowledge of dental problems in Manoor Valley (Northern Himalaya), Pakistan. J. Ethnopharmacol. 2016, 194, 877-894. [CrossRef] [PubMed]

88. Mahapatra, A.K.; Tewari, D.D. Importance of non-timber forest products in the economic valuation of dry deciduous forests of India. For. Pol. Econ. 2005, 7, 455-467. [CrossRef]

89. Khan, F.S.; Islam, M.A.; Gangoo, S.A.; Gatoo, A.A.; Mughal, A.H.; Maqbool, S.; Atta, U. Health care and livelihood support through medicinal plants in indigenous communities of LEH district in Ladakh. J. Pharmacogn. Phytochem. 2018, 7, $1888-1893$. 